\title{
Finite type knot invariants and the calculus of functors
}

\author{
Ismar Volić
}

\begin{abstract}
We associate a Taylor tower supplied by the calculus of the embedding functor to the space of long knots and study its cohomology spectral sequence. The combinatorics of the spectral sequence along the line of total degree zero leads to chord diagrams with relations as in finite type knot theory. We show that the spectral sequence collapses along this line and that the Taylor tower represents a universal finite type knot invariant.
\end{abstract}

\section{Introduction}

In this paper we bring together two fields, finite type knot theory and Goodwillie's calculus of functors, both of which have received considerable attention during the past ten years. Goodwillie and his collaborators have known for some time, through their study of the embedding functor, that there should be a strong connection between the two [GKW01, GW99]. This is indeed the case, and we will show that a certain tower of spaces in the calculus of embeddings serves as a classifying object for finite type knot invariants.

Let $\mathcal{K}$ be the space of framed long knots, i.e. embeddings of $\mathbb{R}$ into $\mathbb{R}^{3}$ which agree with some fixed linear inclusion of $\mathbb{R}$ into $\mathbb{R}^{3}$ outside a compact set, and come equipped with a choice of a nowherevanishing section of the normal bundle. Alternatively, we may think of $\mathcal{K}$ as the space of framed based knots in $S^{3}$, namely framed maps of the unit interval $I$ to $S^{3}$ which are embeddings except at the endpoints. The endpoints are mapped to some basepoint in $S^{3}$ with the same derivative. It is not hard to see that these two versions of $\mathcal{K}$ are homotopy equivalent.

Now consider (framed) singular long knots which are (framed) long knots with a finite number of transverse double points. We can extend any knot invariant $V$ to such knots by applying it to all knots obtained by resolving the double points of a singular knot in two possible ways, with appropriate signs. If $V$ vanishes on knots with more than $n$ double points, then it is of type $n$.

As will be explained in more detail in $\S 2$, it turns out that $\mathcal{V}_{n}$, the set of all type $n$ invariants, is closely related to the space generated by chord diagrams with $n$ chords modulo a certain relation. In fact, if $\mathcal{W}_{n}$ denotes the dual of this space, then Kontsevich [Kon93] proves the following.

THEOREM 1.1. $\mathcal{V}_{n} / \mathcal{V}_{n-1} \cong \mathcal{W}_{n}$.

An alternative proof of this theorem uses Bott-Taubes configuration space integrals, which play a crucial role in the proofs of our results. These are briefly described in $\S 3$, but more details can be found in [BT94, Vol04].

The other ingredient we need is the Taylor tower for $\mathcal{K}$ defined in some detail in $\S 4$. Following the work of Weiss [Wei99], one can construct spaces $\mathcal{H}_{r}$ for each $r>1$, defined as homotopy limits of certain diagrams of 'punctured knots', namely embeddings of $I$ in $S^{3}$ as before but with some number of subintervals removed. There are canonical maps $\mathcal{K} \rightarrow \mathcal{H}_{r}$ and $\mathcal{H}_{r} \rightarrow \mathcal{H}_{r-1}$, and these combine to yield the Taylor tower.

Received 30 January 2004, accepted in final form 31 January 2005.

2000 Mathematics Subject Classification 57R40 (primary), 57M27, 57T35 (secondary).

Keywords: calculus of functors, finite type invariants, Vassiliev invariants, knots, spaces of knots, configuration spaces.

This journal is (c) Foundation Compositio Mathematica 2006. 


\section{Finite TYPE KNOT INVARIANTS AND THE CALCULUS OF FUNCTORS}

To study the tower, we introduce in $\S 5$ a cosimplicial space $X^{\bullet}$ whose $r$ th partial totalization is precisely the stage $\mathcal{H}_{r}$ (Theorem 5.6). This equivalence was shown by Sinha [Sin02]. The advantage of the cosimplicial point of view is that $X^{\bullet}$ comes equipped with a (second quadrant) cohomology spectral sequence. This spectral sequence computes the cohomology of the total complex $T_{r}^{*}$ of the double complex obtained by applying cochains to the truncated cosimplicial space. Alternatively, one can pass to real linear combinations generated by the spaces in the cosimplicial space and take the $r$ th partial totalization to get $T_{r}^{*}$ again. It is the latter description of this algebraic analog of the Taylor tower that we use for our proofs (see $\S 6.3$ for an exact description of the stages $T_{r}^{*}$ ), because sums of resolutions of singular knots can be mapped into such a tower. We can now state the main result.

Theorem 1.2. $H^{0}\left(T_{2 n}^{*}\right) \cong \mathcal{V}_{n}$.

Moreover, all finite type invariants factor through the algebraic Taylor tower for $\mathcal{K}$, as the above isomorphism is induced by a map which is essentially a collection of evaluation maps (see Proposition 5.5). Unfortunately, it is not known that the algebraic stages and ordinary stages of the Taylor tower have the same homology, although this is believed to be the case (Sinha [Sin02] has shown this for any space of embeddings of one manifold in another, as long as the codimension is at least three).

The starting point in the proof of the above theorem is Proposition 6.2, which states that the groups on the diagonal of the $E_{1}$ term of the spectral sequence can be associated to chord diagrams. We then show:

- $E_{\infty}^{-2 n, 2 n} \cong H^{0}\left(T_{2 n}^{*}\right) / H^{0}\left(T_{2 n-1}^{*}\right)(\S 6.2)$;

- $E_{2}^{-2 n, 2 n} \cong \mathcal{W}_{n}$ (Proposition 6.3);

- there is a commutative diagram

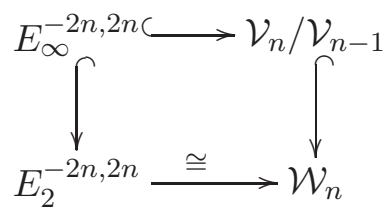

(Proposition 6.4).

The proof of the first statement is a direct computation of the first differential in the spectral sequence. The other parts follow from considering how a singular knot maps into the algebraic Taylor tower.

We then use Theorem 4.2 to finish the proofs in $\S 6.4$. This theorem essentially states that Bott-Taubes integration fits as a diagonal map from $\mathcal{W}_{n}$ to $E_{\infty}^{-2 n, 2 n}$ in the above square and makes the top resulting triangle commute. It follows that all the maps in the diagram are isomorphisms over the rationals (Bott-Taubes integrals are the only reason we cannot state our results over the integers). In addition to deducing Theorem 1.2, we also have an alternative proof of Theorem 1.1, as well as the following.

TheOREM 1.3. The spectral sequence collapses on the diagonal at $E_{2}$.

Thus, the calculus of the embedding functor provides a new point of view on finite type knot theory. Some further questions as well as a brief discussion of the potential importance of this point of view can be found in $\S 6.5$. 


\section{VOLIĆ}

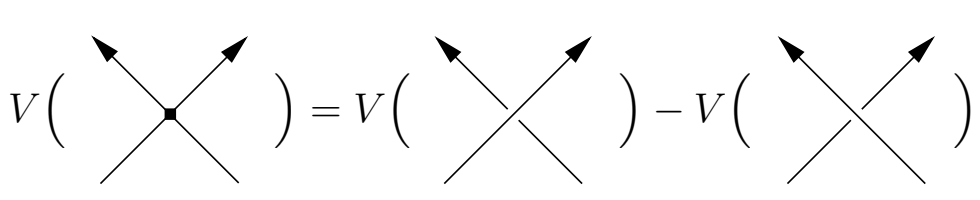

FiguRE 1. Vassiliev skein relation.

\section{Finite type knot invariants}

Here we recall some basic features of finite type knot theory. More details can be found in [Bar95, BS97].

As mentioned earlier, a singular long knot is a long knot except for a finite number of double points. The tangent vectors at the double points are required to be independent. A knot with $n$ such self-intersections is called $n$-singular.

One can now use the Vassiliev skein relation, shown in Figure 1, to extend any knot invariant $V$ to singular knots. The diagrams mean that the three knots only differ locally in one crossing. A $n$-singular knot thus produces $2^{n}$ resolutions. The sign convention ensures that the order in which we resolve the singularities does not matter.

Definition 2.1. $V$ is a (finite or Vassiliev) type $n$ invariant if it vanishes identically on singular knots with $n+1$ self-intersections.

Let $\mathcal{V}$ be the collection of all finite type invariants and let $\mathcal{V}_{n}$ be the set of type $n$ invariants. It is easy to see, for example, that $\mathcal{V}_{0}$ and $\mathcal{V}_{1}$ (for unframed knots) both contain only the constant functions on $\mathcal{K}$. Also immediate is that $\mathcal{V}_{n}$ contains $\mathcal{V}_{n-1}$.

Another quick consequence of the definition is that the value of a type $n$ invariant on an $n$-singular knot only depends on the placement of its singularities. This is because if two $n$-singular knots differ only in the embedding, the difference of $V \in \mathcal{V}_{n}$ evaluated on one and the other is the value of $V$ on some $(n+1)$-singular knots (since one can get from one knot to the other by a sequence of crossing changes). However, by definition $V$ vanishes on such knots.

It thus follows that the value of $V$ on $n$-singular knots is closely related to the following objects.

Definition 2.2. A chord diagram of degree $n$ is an oriented interval with $2 n$ paired-off points on it, regarded up to orientation-preserving diffeomorphisms of the interval.

The pairs of points can be thought of as prescriptions for where the singularities on the knot should occur, while how the rest of the interval is embedded is immaterial.

Let $C D_{n}$ be the set of all chord diagrams with $n$ chords. If $D$ is an element of $C D_{n}$, and if $K_{D}$ is any $n$-singular knot with singularities as prescribed by $D$, by observations above we have a map

$$
\mathcal{V}_{n} \longrightarrow\left\{f: \mathbb{R}\left[C D_{n}\right] \rightarrow \mathbb{R}\right\}
$$

given by

$$
f(D)=V\left(K_{D}\right)
$$

and extending linearly. The kernel is by definition $\mathcal{V}_{n-1}$.

Now let $\mathcal{D}_{n}^{c}=\mathbb{R}\left[C D_{n}\right] / 4 T$, where $4 T$ (or four-term) relation is given in Figure 2. The diagrams differ only in chords indicated; there may be more chords with their endpoints on the dotted segments, but they are the same for all four diagrams. This is a natural relation to impose since it simply reflects the fact that moving a strand of an $(n-1)$-singular knot around one of the singularities gets us back to the original position while introducing four $n$-singular knots on its circular route. 


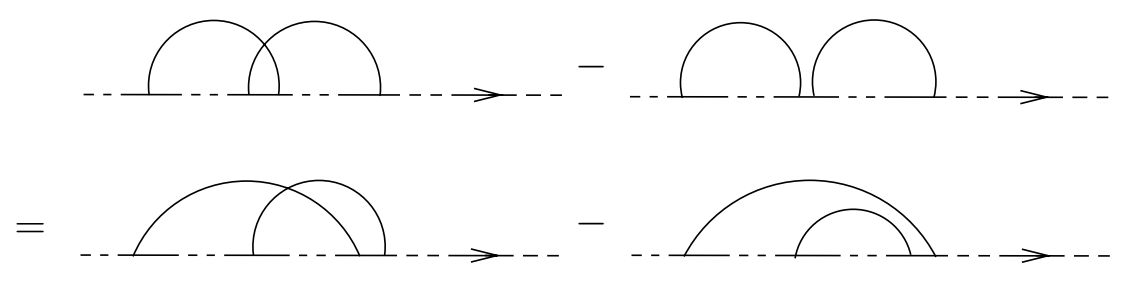

FiguRE 2. $4 T$ relation.

Remark. In most literature on finite type theory, one more relation in addition to the $4 T$ is imposed. This $1 T$ (one-term) relation sets the value of any weight system on a chord diagram with an isolated chord, i.e. a chord not intersected by any other chords, to be zero. However, since we will be considering framed knots, the $1 T$ relation cannot be imposed. This is because the two resolutions of a singularity coming from an isolated chord are not framed isotopic. The consequence of having to consider framed knots is simply that the number of finite type invariants is somewhat larger. One now gets a genuine type 1 invariant, the framing number. The $n$th power of the framing number as well as its product with any type $n-1$ invariant give additional type $n$ invariants.

Let $\mathcal{W}_{n}$, the space of weight systems of degree $n$, be the dual of $\mathcal{D}_{n}^{c}$. It turns out that $\mathcal{W}_{n}$ is all there is to the image of the map in (1). As stated in the introduction, we have the following important theorem due to Kontsevich [Kon93].

THEOREM 2.3. $\mathcal{V}_{n} / \mathcal{V}_{n-1} \cong \mathcal{W}_{n}$.

The inverse of the map in (1) is given by the famous Kontsevich integral, so it is an example of a universal finite type invariant. Alternatively, one can use Bott-Taubes configuration space integrals which we describe in the next section.

\section{Bott-Taubes integrals}

What follows is a brief outline of how configuration space integrals can be used to produce a universal finite type invariant. The integration techniques mentioned here first arose in ChernSimons perturbation theory (see [Lab99] for the history of the theory), but Bott and Taubes [BT94] were the first to present and develop the configuration space integrals in a physics-free way.

Generalizing the computation of the linking number, they consider configurations of $2 n$ points on a knot and use a labeled chord diagram $D$ as a prescription for constructing $n$ maps to the product of 2-spheres.

More precisely, let $F(k, N)$ denote the configuration space of $k$ ordered points in a manifold $N$ :

$$
F(k, N)=\left\{\left(x_{1}, \ldots, x_{k}\right) \in N^{k}, x_{i} \neq x_{j} \text { for } i \neq j\right\} .
$$

Let $K$ be a knot. Given a configuration in $F(2 n, \mathbb{R}), K$ can be evaluated on the $2 n$ points to yield a configuration in $F\left(2 n, \mathbb{R}^{3}\right)$. We denote this map by $e v_{K}$. Now let $p_{i}$ and $p_{j}$ be two points in the image of $e v_{K}$ whose counterparts, $i$ and $j$, are two vertices connected by a chord in $D$. The composition of interest is then

$$
F(2 n, \mathbb{R}) \times \mathcal{K} \stackrel{e v_{K}}{\longrightarrow} F\left(2 n, \mathbb{R}^{3}\right) \stackrel{\frac{p_{j}-p_{i}}{\left|p_{j}-p_{i}\right|}}{\longrightarrow} S^{2} .
$$

There are as many maps to $S^{2}$ as there are chords in $D$. Their product can be used for pulling back $n$ standard unit volume forms from the product of spheres to $F(2 n, \mathbb{R}) \times \mathcal{K}$. 


\section{VOLIĆ}

If one started with two disjoint knots and a configuration space of one point on each of them, the pushforward of the resulting form to the space of disjoint embeddings of two circles would then yield an invariant of 2-component links, the linking number. However, the situation for one knot is not as simple. The configuration space $F(2 n, \mathbb{R})$ is not compact, so that the pushforward to $\mathcal{K}$ may not converge. What is required, as it turns out, is a useful compactification of the configuration spaces appearing in (3).

The first construction of the correct compactification is due to Fulton and MacPherson [FM94] (Bott and Taubes use a modification by Axelrod and Singer [AS94]). At the heart of this compactification is the blowup along each diagonal of the ordered product of $k$ copies of $N$. However, we give here an alternative definition due to Sinha [Sin04b] which does not involve blowups and is thus perhaps more accessible.

Assuming $N$ is embedded in a Euclidean space of dimension $m$, let $i$ be the inclusion of $F(k, N)$ in $N^{k}, \pi_{i j}$ the map to $S^{m-1}$ given by the normalized difference of points $p_{j}$ and $p_{i}$, and $s_{i j k}$ the map to $[0, \infty]$ given by $\left|p_{i}-p_{j}\right| /\left|p_{i}-p_{k}\right|$.

Definition 3.1. Let $F[k, N]$ be the closure of the image of $F(k, N)$ in $N^{k} \times\left(S^{m-1}\right)^{\left(\begin{array}{l}k \\ 2\end{array}\right)} \times[0, \infty]^{\left(\begin{array}{l}k \\ 3\end{array}\right)}$ under the map $i \times \pi_{i j} \times s_{i j k}$.

This compactification is a stratified manifold (manifold with corners), whose stratification is determined by the rates at which configuration points are approaching each other. In particular, a point in a codimension one stratum is determined by some number of configuration points colliding at the same time.

The most useful feature of this construction is that the directions of approach of the colliding points are kept track of. This allows Bott and Taubes to rewrite (3) as

$$
F[2 n, \mathbb{R}] \times \mathcal{K} \longrightarrow F\left[2 n, \mathbb{R}^{3}\right] \longrightarrow S^{2}
$$

and they show that the product of the 2-forms which are pulled back from the spheres extends smoothly to the boundary of $F[2 n, \mathbb{R}]$ (for details, also see [Vol04]). The advantage is that now one can produce a function on the space of knots $\mathcal{K}$ by integrating the resulting $2 n$-form along the compact fiber $F[2 n, \mathbb{R}]$ of the projection

$$
\pi: F[2 n, \mathbb{R}] \times \mathcal{K} \longrightarrow \mathcal{K} .
$$

More precisely, let $\omega$ be the product of the volume forms on $\left(S^{2}\right)^{n}$ and let $h_{D}$ be the product of the compositions in (4). What has just been described is a function on $\mathcal{K}$, which we denote by $I(D, K)$, given by

$$
I(D, K)=\pi_{*}\left(h_{D}^{*} \omega\right) .
$$

The question now is whether $I(D, K)$ is a closed 0 -form, or a knot invariant. To check this, it suffices by Stokes' theorem to examine the pushforward $\pi_{*}$ along the codimension one faces of $F[2 n, \mathbb{R}]$. If the integrals vanish on every such face, (5) yields a knot invariant.

This, however, turns out to be too much to hope for. The boundary integrals along some of the faces are nonzero, and one is next lead to consider other terms to counter their contribution. The correct setting for doing so is provided by first extending the chord diagrams to trivalent diagrams. In addition to chords on the circle, such diagrams have some number of edges ending in triples at vertices off the circle. The diagrams are oriented by one of the two cyclic orientations of the edges emanating from the trivalent vertices (see [Bar95, Definition 1.8]).

Let $T D_{n}$ be the set of trivalent diagrams with $2 n$ vertices. Also consider the $S T U$ relation in Figure 3. Bar-Natan uses the fact that the $4 T$ relation looks like a difference of two such relations to prove the following theorem. 

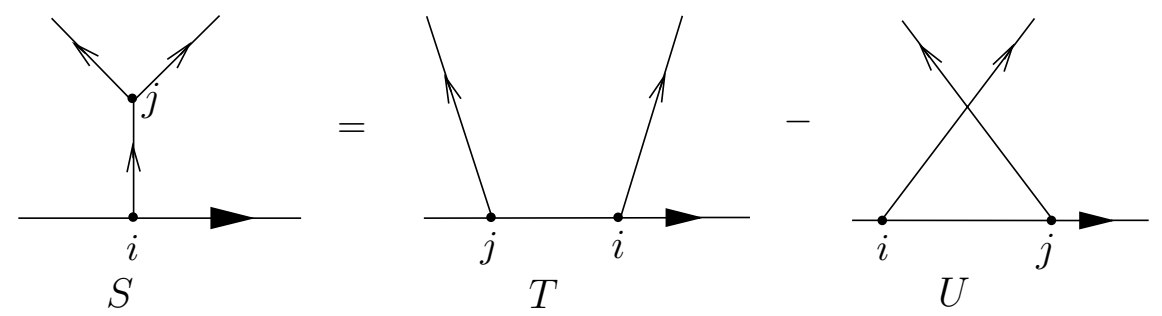

FiguRE 3. STU relation.

Theorem 3.2 [Bar95, Theorem 6]. The real vector space generated by trivalent diagrams with $2 n$ vertices modulo the $S T U$ relation is isomorphic to $\mathcal{D}_{n}^{c}$.

The weight systems now extend uniquely to trivalent diagrams modulo $S T U$.

Given a trivalent diagram with $2 n$ vertices, $k$ of which are on the circle and $s$ are trivalent, Bott and Taubes now construct a $(k+3 s)$-dimensional space $F\left[k, s ; \mathcal{K}, \mathbb{R}^{3}\right]$ which fibers over $\mathcal{K}$ with fiber a configuration space of $2 n$ points in $\mathbb{R}^{3}$ with the condition that $k$ of these points are constrained to lie on a given knot $K$. Fulton-MacPherson compactification may also be carried out for these spaces. Since trivalent diagrams are generalizations of chord diagrams, $F\left[k, s ; \mathcal{K}, \mathbb{R}^{3}\right]$ reduces to $F\left[2 n, S^{1}\right] \times \mathcal{K}$ when $s=0$.

So now trivalent diagrams $D$ become prescriptions for pulling back 2-forms from products of spheres. Each chord and edge of $D$ again determines a map to $S^{2}$ via the normalized difference of the appropriate two points in $F\left[k, s ; \mathcal{K}, \mathbb{R}^{3}\right]$, and each map is used to pull back a volume form from a sphere. As described in [BT94, Vol04], the resulting form pulled back by the product of all the maps extends smoothly to the codimension one strata of $F\left[k, s ; \mathcal{K}, \mathbb{R}^{3}\right]$. Since $D$ has $(k+3 s) / 2$ chords and edges, the resulting form on $F\left[k, s ; \mathcal{K}, \mathbb{R}^{3}\right]$ is $(k+3 s)$-dimensional. Its pushforward thus again yields a function on $\mathcal{K}$.

Let $I(D, K)$ denote the pullback followed by pushforward as before, only now $D$ could also be a trivalent diagram. The following theorem was proved by Altschuler and Freidel [AF95], while a different partial proof was given by Thurston [Thu95] following the work of Bar-Natan and Bott-Taubes (see also [Vol04]).

Theorem 3.3. Let $W$ be a weight system in $\mathcal{W}_{n}$. The sum of $I(D, K)$ with coefficients $W(D)$, taken over all trivalent diagrams $D$ with $2 n$ vertices and with a certain correction term for each $D$, is a knot invariant. Further, when $I(D, K)$ is extended to sums of resolutions of singular knots, it gives a universal type $n$ invariant.

The proof is a combination of arguments showing that the integrals along the various faces of $F\left[k, s ; \mathcal{K}, \mathbb{R}^{3}\right]$ either vanish, cancel due to the relations imposed on trivalent diagrams, or can be compensated for by other integrals. Next we will see how this theorem extends to the stages of the Taylor tower for $\mathcal{K}$.

\section{Taylor tower for the space of knots}

Building on the general theory of the calculus of functors developed by Goodwillie [Goo90, Goo92, Goo93], Weiss develops in [Wei99] (also see [GKW01]) a certain tower for studying the space of embeddings of one manifold in another. The stages of the tower in some sense approximate the original space of embeddings as long as the codimension of one manifold in the other is at least three. 


\section{VOLIĆ}

This is of course not true in our situation, but the tower for $\mathcal{K}$ can still be constructed as outlined below. More on this construction for the particular case of spaces of knots can be found in [Sin02, Vol04].

Let $\left\{A_{i}\right\}, 1 \leqslant i \leqslant r$, be a collection of disjoint closed subintervals of $I$ not containing the endpoints, and define spaces of 'punctured knots'

$$
E_{S}=\operatorname{Emb}\left(I-\bigcup_{i \in S} A_{i}, S^{3}\right)
$$

for each nonempty subset $S$ of $\{1, \ldots, r\}$. Here we as usual mean that the endpoints of the interval are sent to some basepoint in $S^{3}$ with the same derivative while the other pieces of $I$ are embedded.

These spaces can be arranged in a subcubical diagram (like a cubical diagram, but missing a space which maps to all others), which we will call $E C_{r}$, since every punctured knot restricts to a knot with more punctures. Thus, for example, if $r=3$, we have a 3-subcubical diagram $E C_{3}$.

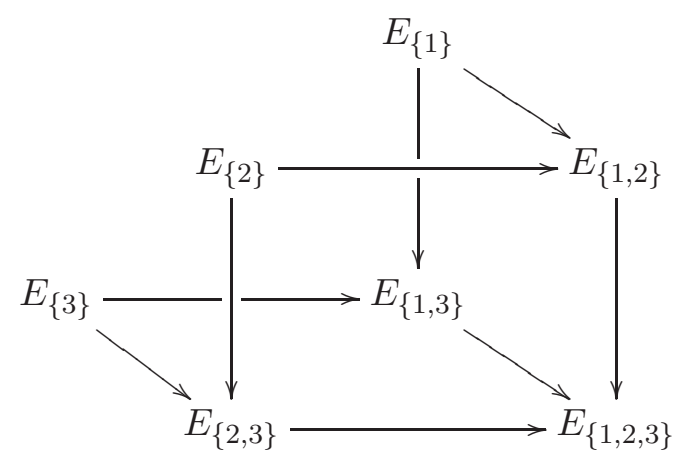

Each square side of such a diagram commutes since the order in which the subintervals of $I$ are removed does not matter.

Now let $\Delta^{S}$ be the face of $\Delta^{r-1}$ for which those barycentric coordinates indexed by elements of $\{1, \ldots, r\}$ which are not in the subset $S$ are 0 . Recall that the homotopy limit of $E C_{r}$, which we will denote by $\mathcal{H}_{r-1}$, can then be defined as the subspace of

$$
\prod_{\emptyset \neq S \subseteq\{1, \ldots, r\}} \operatorname{Maps}\left(\Delta^{S}, E_{S}\right)
$$

consisting of collections of maps $\left\{\alpha_{S}\right\}$ such that, for every map $E_{S} \rightarrow E_{S \cup\{i\}}$ in the diagram, the square

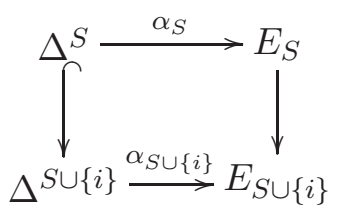

commutes. (More on homotopy limits of diagrams of spaces can be found in [BK72, Goo92].)

Two observations are immediate. Any knot can be restricted to a knot with punctures, so that $\mathcal{K}$ maps to the subcubical diagram (in fact, $\mathcal{K}$ is also the actual limit of the subcubical diagram, as long as $r>2$ ). Consequently, there are canonical maps $\mathcal{K} \longrightarrow \mathcal{H}_{r}$. Also, the subcubical diagram for knots with up to $r$ punctures is contained in the diagram for knots with up to $r+1$ punctures, so that there are also projections $\mathcal{H}_{r} \longrightarrow \mathcal{H}_{r-1}$.

Definition 4.1. The Taylor tower for $\mathcal{K}$ is the collection of maps

$$
\mathcal{H}_{1} \longleftarrow \cdots \longleftarrow \mathcal{H}_{r-1} \longleftarrow \mathcal{H}_{r} \longleftarrow \cdots .
$$

Spaces $\mathcal{H}_{r}$ will be called the stages of the tower. 


\section{FinITE TYPE KNOT INVARIANTS AND THE CALCULUS OF FUNCTORS}

Remark. In the most general setting, the Taylor tower is constructed from embeddings of codimension zero submanifolds of one manifold in another. As mentioned earlier, if the codimension is at least three, Goodwillie, Klein and Weiss [GK05, GW99] show that the maps from the original space of embeddings to stages of the tower induce more isomorphisms on homotopy and homology groups the higher one goes in the tower. Consequently, the inverse limit of the tower is weakly equivalent to the original space of embeddings. In particular, this fact has been used for studying the homology and homotopy of spaces of knots in $\mathbb{R}^{m}, m>3$ (see [LV05, SS02, Sin02, Vol04]).

In [Vol04], we show how Bott-Taubes integrals can be extended to the stages. We integrate over certain spaces which generalize $F\left[k, s ; \mathcal{K}, \mathbb{R}^{3}\right]$. The $k$ points no longer represent a configuration on a single knot, but rather on a family of punctured knots $h$, i.e. an element of $\mathcal{H}_{2 n}$. Denoting by $I(D, h)$ the analog of $(5)$, we prove the following.

Theorem 4.2 [Vol04, Theorem 4.5]. Let $W$ be a weight system in $\mathcal{W}_{n}$ and let $D$ be a trivalent diagram with $2 n$ vertices. Then the sum of $I(D, h)$ over all $D$, with coefficients $W(D)$, is an invariant of $\mathcal{H}_{2 n}$, provided a correction term is given for each $D$. In particular, this restricts to the invariant of $\mathcal{K}$ described in Theorem 3.3 when a point in $\mathcal{H}_{2 n}$ comes from a knot.

The next goal is to see how our invariants $T(W)$ behave when singular knots, or rather sums of their resolutions, are mapped into the stages $\mathcal{H}_{2 n}$. To that end, we introduce a cosimplicial model for the Taylor tower because it is suitable for our computational purposes.

\section{A cosimplicial model for the Taylor tower}

\subsection{A cosimplicial space of configurations in a manifold}

Let $M$ be a simply connected manifold of dimension at least three, embedded in $\mathbb{R}^{d}$ for some large $d$. We will later specialize to $M=S^{3}$. Let

$$
\begin{gathered}
p=\left(p_{0}, p_{1}, \ldots, p_{r}, p_{r+1}\right), \quad p_{i} \in M \\
v=\left(v_{i j}: 0 \leqslant i \leqslant j \leqslant r+1\right), \quad v_{i j} \in V,\left|v_{i j}\right|=1 .
\end{gathered}
$$

Let $b$ be some fixed point in $M$ and $\beta$ a fixed unit vector in $T_{b} M$, the tangent space of $M$ at $b$. Define

$$
Y^{r} \subset M^{r+2} \times\left(S^{d-1}\right)\left(\begin{array}{c}
r+2 \\
2
\end{array}\right)
$$

with subspace topology as follows- $(p, v)$ is in $Y^{r}$ if it satisfies:

(1) $p_{0}=p_{r+1}=b$;

(2) $v_{i i} \in T_{p_{i}} M, v_{00}=v_{(r+1)(r+1)}=\beta$;

(3) $p_{i}=p_{j} \Longrightarrow\left\{\begin{array}{l}p_{i}=p_{k}, \quad v_{i i}=v_{k l}=v_{j j}, \quad i \leqslant k \leqslant l \leqslant j ; \\ \text { or } \\ p_{0}=p_{1}=\cdots=p_{i}, \quad v_{00}=v_{k l}=v_{i i}, \quad 0 \leqslant k \leqslant l \leqslant i \\ \text { and } \\ p_{j}=p_{j+1}=\cdots=p_{r+1}, \quad v_{j j}=v_{m n}=v_{(r+1)(r+1)}, \quad j \leqslant m \leqslant n \leqslant r+1 .\end{array}\right.$

Let $U^{r}=\{(p, v)\} \in M^{r} \times\left(S^{d-1}\right)^{r}$ with

$$
\begin{gathered}
p=\left(p_{1}, p_{2}, \ldots, p_{r}\right), \quad p_{i} \neq p_{j}, p_{i} \neq b, \text { for all } i, j \\
v=\left(v_{1}, v_{2}, \ldots, v_{r}\right), \quad v_{i} \in T_{p_{i}} M,\left|v_{i}\right|=1 .
\end{gathered}
$$




\section{VOLIĆ}

Thus, $U^{r}$ is the space of configurations of $r$ distinct points in $M \backslash\{b\}$ labeled with tangent vectors. In particular, if the dimension of $M$ is $d$,

$$
U^{r}=F(r, M \backslash\{b\}) \times\left(S^{d-1}\right)^{r}
$$

This space maps to $Y^{r}$ by

$$
\left(p_{1}, \ldots, p_{r}, v_{1}, \ldots, v_{r}\right) \longmapsto\left(b, x_{1}, \ldots, x_{r}, b, v_{i j}\right)
$$

where

$$
v_{i j}= \begin{cases}v_{i}, & \text { if } 0<i=j<m+1, \\ \beta, & \text { if } i=j=0 \text { or } i=j=m+1, \\ \frac{p_{j}-p_{i}}{\left|p_{j}-p_{i}\right|}, & \text { if } i<j .\end{cases}
$$

It is easy to see that this map is one-to-one and a homeomorphism onto its image, so $U^{r}$ can be identified with a subspace of $Y^{r}$.

Definition 5.1. Let $X^{0}=(b, b, \beta)$ and define $X^{r}$ for all $r>0$ to be the closure of $U^{r}$ in $Y^{r}$.

We now define the coface and codegeneracy maps, $\partial^{i}$ and $s^{i}$, which will be given by doubling and forgetting points and vectors.

Let

$$
\partial^{i}: Y^{r} \longrightarrow Y^{r+1}
$$

be given by

$$
\left(p_{1}, \ldots, p_{i}, \ldots, p_{r}, v_{i j}\right) \longmapsto\left(p_{0}, \ldots, p_{i}, p_{i}, \ldots, p_{r+1}, v_{i j}^{\prime}\right),
$$

where the $v_{i j}$ map to $v_{i j}^{\prime}$ by

$$
\left(\begin{array}{ccccc}
\ddots & \vdots & \vdots & \vdots & \vdots \\
& v_{i i} & v_{i(i+1)} & \ldots & v_{i(r+1)} \\
& & \ddots & \vdots & \vdots
\end{array}\right) \longmapsto\left(\begin{array}{ccccccc}
\ddots & \vdots & \vdots & \vdots & \vdots & \vdots \\
& & v_{i i} & v_{i i} & v_{i(i+1)} & \ldots & v_{i(r+1)} \\
& & v_{i i} & v_{i(i+1)} & \ldots & v_{i(r+1)} \\
& & & \ddots & \vdots & \vdots
\end{array}\right) .
$$

Here one should keep in mind that $p_{0}=p_{r+1}=b, v_{00}=v_{(r+1)(r+1)}=\beta$ as before, and also that $v_{(i+1)(i+1)}=v_{i(i+1)}=v_{i i}$ in the image array. The following is immediate from the definitions.

LEMMA 5.2. $\partial^{i}\left(X^{r}\right) \subset X^{r+1}$.

This lemma is true for $x_{i}=x_{0}=b$ or $x_{i}=x_{r+1}=b$, so that we indeed get all the doubling maps we want:

$$
\partial^{i}: X^{r} \longrightarrow X^{r+1}, \quad 0 \leqslant i \leqslant r+1 .
$$

For $s^{i}$, the forgetting maps, the situation is much simpler. Define

$$
s^{i}: X^{r+1} \longrightarrow X^{r}, \quad 1 \leqslant i \leqslant r
$$

by omitting the entry $p_{i}$ as well as all vectors for which at least one index is $i$, and then relabeling the result. This is clearly continuous.

Proposition 5.3. Let $X^{\bullet}$ be the sequence of spaces $X^{0}, X^{1}, X^{2}, \ldots$, together with doubling and forgetting maps $\partial^{i}$ and $s^{i}$ as given above. Then $X^{\bullet}$ is a cosimplicial space.

The proof is a straightforward check of the cosimplicial identities and is left to the reader. 


\section{Finite TYPE KNOT INVARIANTS AND THE CALCULUS OF FUNCTORS}

\subsection{Equivalence of totalizations and stages of the Taylor tower}

Recall that $\Delta^{\bullet}$ is the cosimplicial space made of closed simplices $\Delta^{r}$, with cofaces and codegeneracies the inclusions of and projections onto faces.

Also recall that the totalization $\operatorname{Tot}^{\bullet}$ of a cosimplicial space $X^{\bullet}$ is a subspace of the space of maps from $\Delta^{\bullet}$ to $X^{\bullet}$, and can be defined as

$$
\lim _{\longleftarrow}\left(\operatorname{Tot}^{1} X^{\bullet} \longleftarrow \operatorname{Tot}^{2} X^{\bullet} \longleftarrow \cdots\right) .
$$

Here $\operatorname{Tot}^{r} X^{\bullet}$ is the rth partial totalization, or the subspace of

$$
\prod_{0 \leqslant i \leqslant r} \operatorname{Maps}\left(\Delta^{i}, X^{i}\right), \quad 0 \leqslant i \leqslant r
$$

determined by the compatibility condition that the squares

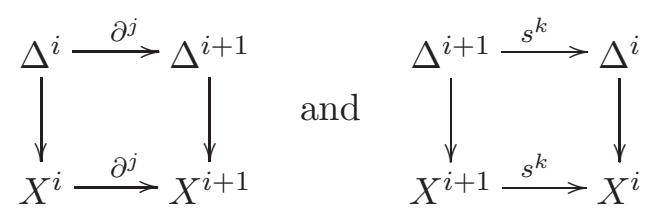

commute for all cofaces $\partial^{j}$ and codegeneracies $s^{k}$.

We now consider the special case $M=S^{3}$. Recall that $K \in \mathcal{K}$ is a map of $I$ in $S^{3}$ embedding the interior and sending 0 and 1 to a fixed point $b \in S^{3}$ with the same fixed tangent vectors $K^{\prime}(0)=K^{\prime}(1)=\beta$. Let $\Delta^{r}$ be parametrized by $\left(0=x_{0}, x_{1}, \ldots, x_{r}, x_{r+1}=1\right)$.

Definition 5.4. Given a knot $K$, define

$$
e v_{r}(K): \Delta^{r} \longrightarrow X^{r}
$$

by

$$
e v_{r}(K)\left(x_{0}, x_{1}, \ldots, x_{r}, x_{r+1}\right)=\left(b, K\left(x_{1}\right), \ldots, K\left(x_{r}\right), b, v_{i j}\right)
$$

where

$$
v_{i j}= \begin{cases}\frac{K^{\prime}\left(x_{i}\right)}{\left|K^{\prime}\left(x_{i}\right)\right|}, & \text { if } 0<i=j<r+1, \\ \frac{K\left(x_{j}\right)-K\left(x_{i}\right)}{\left|K\left(x_{j}\right)-K\left(x_{i}\right)\right|}, & \text { if } 0 \leqslant i \neq j<r+1 \text { or } 0<i \neq j \leqslant r+1 .\end{cases}
$$

Let

$$
e v_{r}: \mathcal{K} \longrightarrow \operatorname{Maps}\left(\Delta^{r}, X^{r}\right)
$$

be defined by sending $K$ to $e v_{r}(K)$.

It is clear that $e v_{r}(K)$ is a continuous map to $X^{r}$ since it is continuous into $Y^{r}$ and it takes $\operatorname{int}\left(\Delta^{r}\right)$ into $U^{r}$. The following is a straightforward check of the compatibility of $e v_{r}$ with the cosimplicial maps.

Proposition 5.5. Denote by $e v_{[r]}$ the collection of maps ev,$l \leqslant r$. Then $e v_{[r]}$ maps $\mathcal{K}$ to $\operatorname{Tot}^{r} X^{\bullet}$.

We may therefore arrange the maps between partial totalizations of $X^{\bullet}$ and the maps they admit from $\mathcal{K}$ into a tower much like the Taylor tower in (6), except with $\mathcal{H}_{r}$ replaced by $T_{0} t^{r} X^{\bullet}$. In fact, the next statement says that $X^{\bullet}$ is a good substitute for the Taylor tower.

TheOREm 5.6 [Sin02, Theorem 6.1]. The $r$ th stage $\mathcal{H}_{r}$ of the Taylor tower for $\mathcal{K}$ is weakly equivalent to $\operatorname{Tot}^{r} X^{\bullet}$ for all $r>0$. 


\section{VOLIĆ}

The cosimplicial space Sinha uses in [Sin02] is slightly different from ours, with $X^{r}$ defined as the closure of the image of the configuration space of $r$ points in $S^{3}$ under the map $i \times \pi_{i j}$ (see Definition 3.1). Cofaces and codegeneracies are doubling and forgetting maps like ours. Using Corollary 5.9 and [Sin02, Theorem 4.2], it is easy to see that the two cosimplicial spaces are equivalent. Namely, they both consist of configuration spaces labeled with tangent vectors up to homotopy, and there are obvious equivalences between those spaces respecting the cosimplicial maps. The advantage of our definition is that it is made with knots and evaluation maps in mind (points moving along a knot can only collide in one direction).

We next prove in Proposition 5.7 and Corollary 5.9 that $E_{S}$ (spaces of punctured knots) and $X^{r}$ are configurations labeled with tangent vectors. (The above theorem then in effect says that these configuration spaces are 'put together the same way' in the homotopy limits and partial totalizations. The main observations and tools in Sinha's proof are that the restriction maps between punctured knots look like doubling maps up to homotopy and that a truncated cosimplicial space can be redrawn as a subcubical diagram whose homotopy limit is equivalent to the original totalization. Sinha thus constructs equivalences going through an auxiliary tower whose stages are homotopy limits of cubical diagrams of compactified configuration spaces with doubling maps between them.)

Recall that $E_{S}$ is the space of embeddings of the complement of $s=|S|$ closed subintervals in $S^{3}$, where $S$ is a nonempty subset of $\{1, \ldots, r\}$. In other words, each point in $E_{S}$ is an embedding of $s+1$ open subintervals (or half-open, in the case of the first and last subinterval), which we index in order from 1 to $s+1$.

For each $S$ and each open subinterval, choose points $x_{i}, 1 \leqslant i \leqslant s-1$, in the interiors. The choices should be compatible, namely if $S \subset T$, then $\left\{x_{i}\right\}_{i \in S} \subset\left\{x_{i}\right\}_{i \in T}$. Also let $x_{0}$ and $x_{s}$ be the left and right endpoints of $I$, respectively.

Proposition 5.7. $E_{S} \simeq F\left(s-1, S^{3} \backslash\{b\}\right) \times\left(S^{2}\right)^{s-1}$.

Proof. To specify an embedding at a point, it is sufficient and necessary to specify the image of the point as well as the nonzero derivative of the embedding at that point. For more points, the images additionally must be distinct. Letting $e$ be an embedding in $E_{S}$, we thus have an evaluation map

$$
\begin{aligned}
e v_{S}: E_{S} & \longrightarrow F\left(s-1, S^{3} \backslash\{b\}\right) \times\left(S^{2}\right)^{s-1} \\
e & \longmapsto\left(e\left(x_{0}\right), \ldots, e\left(x_{s}\right), \frac{e^{\prime}\left(x_{0}\right)}{\left|e^{\prime}\left(x_{0}\right)\right|}, \ldots, \frac{e^{\prime}\left(x_{s}\right)}{\left|e^{\prime}\left(x_{s}\right)\right|}\right) .
\end{aligned}
$$

Here we need to remember that the endpoints always map to the origin with the same tangent vector. (As a matter of fact, we could have omitted either one of the endpoints from the above map.)

To see that $e v_{S}$ is an equivalence, observe that its fiber consists of all embeddings in $E_{S}$ going through $s-1$ distinct points in $S^{3}$ with some specified tangent vectors. However, this is a contractible space as any such embedding can be 'shrunk back' to the specified points while preserving the tangent vectors at those points.

We next prove an almost identical statement for the spaces in $X^{\bullet}$ as a corollary of the following. Proposition 5.8. The inclusion $U^{s} \hookrightarrow X^{s}$ induces a weak homotopy equivalence.

Since $U^{s}$ is a space of $s$ configuration points in the interior of $S^{3} \backslash\{b\}$ with vectors attached, we deduce the following.

Corollary 5.9. $X^{s} \sim F\left(s, S^{3} \backslash\{b\}\right) \times\left(S^{2}\right)^{s}$.

To prove Proposition 5.8, we need the following technical result, whose proof is straightforward. 


\section{Finite TYPE KNOT INVARIANTS AND THE CALCULUS OF FUNCTORS}

Lemma 5.10. Let $U$ be an open subset of a space $X$. Suppose

$$
H: X \times[0,1] \longrightarrow X
$$

is a map such that, for each $p \in X, H(p, 0)=p$ and there is a neighborhood $V_{p}$ of $p$ and $\epsilon_{p} \in(0,1]$ with

$$
H(y, t) \in U \quad \text { for all }(y, t) \in\left(V_{p},\left(0, \epsilon_{p}\right]\right) .
$$

Then the inclusion $U \hookrightarrow X$ induces a weak equivalence.

Proof of Proposition 5.8. We wish to define a homotopy

$$
H: X^{s} \times[0,1] \longmapsto X^{s}
$$

satisfying the conditions of the previous lemma, namely that each point $p \in X^{s}$ has a neighborhood $V_{p}$ on which $H$ is a homotopy into $U^{s}$ for some subinterval $\left[0, \epsilon_{p}\right]$. To do this, we use the vectors $v_{i i}$ to 'separate' points $p_{i}$ and $p_{i+1}$ when $p_{i}=p_{i+1}$, thereby mapping them from the boundary of $X^{s}$ to its interior, $U^{s}$.

We first do this locally. For each $p=\left(p_{0}, \ldots, p_{s+1}\right)$ let

$$
w_{i}=\sum_{i=0}^{s+1} c_{i}(p) v_{i i},
$$

where each $c_{i}(p)$ assigns a real number to point $p_{i}$, and they together satisfy the conditions

$$
\left(c_{i+1}-c_{i}\right)(p)>0 \quad \text { if } p_{i}=p_{i+1}, \quad \text { and } \quad c_{0}=c_{s+1}=0 .
$$

Note that, if $p_{j} \neq p_{j+1},(p, v)$ always has a neighborhood in $X^{s}$ such that for all $(y, v)$ in that neighborhood, $y_{j} \neq y_{j+1}$. We therefore also have a neighborhood $V_{p}$ of $(p, v)$ on which $H$, defined as

$$
H\left(p_{i}, v_{i j}, t\right)=\left(p_{i}+t w_{i}, v_{i j}\right),
$$

is a homotopy into $U^{s}$ for at least some time interval $\left[0, \epsilon_{p}\right]$. Further, $c_{i}$ can then be chosen to be constant on $V_{p}$, and if $p_{j} \neq p_{j+1}, c_{j}$ may be set to be 0 so that $H$ is continuous on $V_{p}$.

We may now cover $X^{s}$ with open sets $\left\{V_{\alpha}\right\}$ determined by $V_{p}$, and use a partition of unity to define a continuous $H$ on all of $X^{s}$. It is immediate that the conditions (10) are satisfied on the intersections of the $V_{\alpha}$.

\subsection{Tangential data and framed knots}

To make future arguments work out easier, in this section we remove the tangential data appearing in Proposition 5.7 and Corollary 5.9. The price we will have to pay is that the space of ordinary knots will become a space which on $\pi_{0}$ looks like the space of framed knots whose framing number is even. This is why we worked with framed knots throughout the previous sections, and, as explained earlier, the class of chord diagrams was larger for us than it would have been had the framing not been considered.

First note that $\S 4$ could be repeated with 'immersions' instead of 'embeddings'. So let:

- Im be the space of immersions of $I$ in $S^{3}$ with usual conditions on endpoints;

- $\operatorname{Im}_{S}$ be the space of immersions of $I$ with $s=|S|$ subintervals removed;

- $I m C_{r}$ be the subcubical diagram obtained by considering $\operatorname{Im}_{S}$ for all nonempty subsets $S$ of $\{1, \ldots, r+1\}$ with restriction maps between them; and

- $\operatorname{holim}\left(\operatorname{Im} C_{r}\right)$ be its homotopy limit. 


\section{VOLIĆ}

Recall that Im is homotopy equivalent to $\Omega S^{2}$ since immersions are determined by their derivatives. Similarly, in analogy with Proposition 5.7, we have

$$
\operatorname{Im}_{S} \simeq\left(S^{2}\right)^{s-1} .
$$

The configuration space is no longer present in the equivalence because of the lack of the injectivity condition for immersions.

Before we prove two useful statements, we need the following propositions.

Proposition 5.11 [Goo92, Proposition 1.6]. Suppose $X_{\emptyset}$ completes a subcubical diagram $C_{r}$ of spaces $X_{S}$ indexed by nonempty subsets of $\{1, \ldots, r\}$ into a cubical diagram. If, for every $S$,

$$
X_{S} \longrightarrow \operatorname{holim}\left(X_{S \cup\{j\}} \rightarrow X_{S \cup\{i, j\}} \leftarrow X_{S \cup\{i\}}\right)
$$

is a weak equivalence, then $X_{\emptyset} \rightarrow \operatorname{holim}\left(C_{r}\right)$ is also a weak equivalence.

Proposition 5.12. The map $\operatorname{Im} \longrightarrow \operatorname{holim}\left(\operatorname{Im} C_{r}\right)$ is a weak equivalence.

Proof. Unlike for spaces of punctured embeddings, $I m_{S}$ is the limit of

$$
\operatorname{Im}_{S \cup\{j\}} \longrightarrow \operatorname{Im}_{S \cup\{i, j\}} \longleftarrow \operatorname{Im}_{S \cup\{i\}} \cdot
$$

Further, the map $I m_{S} \rightarrow I m_{S \cup\{i\}}$ is a fibration for all $i$. It follows that $I m_{S}$ is weakly equivalent to the homotopy limit of diagram (12). This holds for any square in $\operatorname{Im} C_{r}$. In addition, we can complete the subcube $\operatorname{Im} C_{r}$ by adding $\operatorname{Im}$ as the initial space. For the extra square diagrams produced this way, we again have that $I m$ is equivalent to their homotopy limits. Proposition 5.11 then finishes the proof.

Proposition 5.13. There is a map of homotopy limits

$$
\operatorname{holim}\left(E C_{r}\right) \longrightarrow \operatorname{holim}\left(\operatorname{Im} C_{r}\right),
$$

whose homotopy fiber is the homotopy limit of a subcubical diagram $F C_{r}$ of spaces which are homotopy equivalent to configuration spaces of up to $r$ labeled points in $S^{3} \backslash\{b\}$.

Proof. Since every embedding is an immersion, there are inclusions $E_{S} \longrightarrow \operatorname{Im}_{S}$ for each $S$. By Proposition 5.7 and (11) this map is, up to homotopy, the projection

$$
F\left(s, S^{3} \backslash\{b\}\right) \times\left(S^{2}\right)^{s} \longrightarrow\left(S^{2}\right)^{s},
$$

with fiber the configuration space $F\left(s, S^{3} \backslash\{b\}\right)$. However, for each $S$ such that $|S|=s$, we get a different configuration space of labeled points in $S^{3} \backslash\{b\}$. It follows that there is a map of subcubes,

$$
E C_{r} \longrightarrow \operatorname{Im} C_{r}
$$

with homotopy fiber a subcubical diagram $F C_{r}$ of configuration spaces as desired.

If we denote by $E$ the space of unframed knots and by $\overline{\mathcal{K}}$ the homotopy fiber of the projection $E \rightarrow I m$, the previous two propositions can be summarized in the diagram

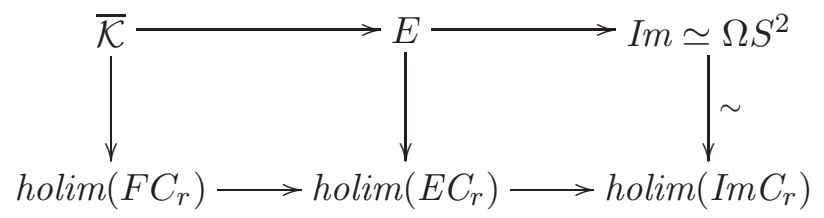

where the left spaces are the homotopy fibers of the two right horizontal maps.

The middle column in the diagram represents the spaces and maps we would have originally considered, but since we wish to remove the tangent spheres, we now shift our attention to the left column. Since the map $E \rightarrow I m$ is null-homotopic [Sin04a, Proposition 5.1], we have

$$
\overline{\mathcal{K}} \simeq E \times \Omega I m
$$




\section{Finite TYPE KNOT INVARIANTS AND THE CALCULUS OF FUNCTORS}

Proposition 5.14. We may identify $\pi_{0}(\overline{\mathcal{K}})$ with the isotopy classes of framed knots whose framing number is even.

Proof. The space of framed knots up to isotopy is $\pi_{0}(E) \times \mathbb{Z}$ since every knot can be framed to yield any framing number and this number is an isotopy invariant. To prove the proposition, it hence suffices to exhibit a bijection

$$
\pi_{0}(\overline{\mathcal{K}}) \stackrel{f}{\longrightarrow} \pi_{0}(E) \times 2 \mathbb{Z} .
$$

A point in $\overline{\mathcal{K}}$ consists of an embedding $\widetilde{K}$ along with a path $\alpha$ from $\widetilde{K}$ through immersions to the basepoint in $I m$, namely the unknot. This path produces a possibly singular surface where the singularities come from $\widetilde{K}$ passing through itself. The standard framing of the unknot (a copy of the unknot, displaced slightly in one direction so that the framing number of the unknot is 0) can be used to obtain a framing of $\widetilde{K}$ by dragging the framing of the unknot along $\alpha$. However, as $\alpha$ goes through a singularity, the framing number of a knot changes by two. It follows that $\widetilde{K}$ must have an even framing number.

Next we have an exact sequence of homotopy groups whose end is

$$
\cdots \rightarrow \mathbb{Z} \rightarrow \pi_{0}(\overline{\mathcal{K}}) \rightarrow \pi_{0}(E) \rightarrow 0 .
$$

Here we have used $\pi_{0}\left(\Omega S^{2}\right)=0$ and $\pi_{1}\left(\Omega S^{2}\right)=\mathbb{Z}$.

Now $\pi_{1}(\operatorname{Im})=\mathbb{Z}$ acts on $\pi_{0}(\overline{\mathcal{K}})$ and it does so in such a way that the action of 1 adds two to the framing number, while the action of -1 subtracts two. This is because the generator for $\pi_{1}(\operatorname{Im})$ is the loop of the unknot that introduces a twist (the first Reidemeister move), passes the crossing obtained that way through itself, and then untwists the result having changed the framing number by two. Depending on the orientation of the unknot and on the direction of the twist (right- or left-handed), this loop corresponds to one of the generators of $\mathbb{Z}$.

Given an element $(K, 2 n)$ in $\pi_{0}(E) \times 2 \mathbb{Z}$, where $K$ represents a knot type, there is a class represented by $(K, \alpha)$ in $\pi_{0}(\overline{\mathcal{K}})$ with $\alpha$ a path of $K$ to the unknot through immersions. The path then provides some framing of $K$ with the framing number $2 m$ so that the action of $n-m$ on $(K, \alpha)$, composed with $f$, gives $(K, 2 n)$. So $f$ is surjective.

Now suppose that two representatives of classes in $\pi_{0}(\overline{\mathcal{K}}),\left(\widetilde{K}_{1}, \alpha_{1}\right)$ and $\left(\widetilde{K}_{2}, \alpha_{2}\right)$, give isotopic knots $K_{1}$ and $K_{2}$ with the same even framing number. By exactness of the homotopy sequence, there exists an integer which acts on $\left(\widetilde{K}_{1}, \alpha_{1}\right)$ to produce $\left(\widetilde{K}_{2}, \alpha_{2}\right)$. However, since $K_{1}$ and $K_{2}$ have the same framing number, this integer must be 0 , so that $\left(\widetilde{K}_{1}, \alpha_{1}\right)$ and $\left(\widetilde{K}_{2}, \alpha_{2}\right)$ represent the same element of $\pi_{0}(\widetilde{E})$. Thus, $f$ is injective.

To set the notation for the next section, let

$$
T_{r}=\operatorname{holim}\left(F C_{r+1}\right),
$$

We thus have the modified Taylor tower for the space of knots,

$$
T_{2} \longleftarrow T_{3} \longleftarrow \cdots \longleftarrow T_{r} \longleftarrow \cdots
$$

where each space is equipped with a map from $\overline{\mathcal{K}}$ (a collection of compatible evaluation maps) making all the resulting triangles commute.

We can now do the same for the cosimplicial space $X^{\bullet}$ and see that Theorem 5.6 is preserved. Namely, since each $X^{s}$ in $X^{\bullet}$ is equivalent to $F\left(s, S^{3} \backslash\{b\}\right) \times\left(S^{2}\right)^{s}$, we consider the cosimplicial space $Y^{\bullet}$ with only $\left(S^{2}\right)^{s+1}$ as its $s$ th space. The maps are inclusions and projections. Then $\operatorname{Tot}^{r} Y^{\bullet}$ is $\Omega S^{2}$ for all $r>1$ (since $Y^{\bullet}$ is just the space of maps from the standard simplicial model for $S^{1}$ to $S^{2}$ ). This in fact allows us to say that there is a tower

$$
\operatorname{Tot}^{2} F\left(s, S^{3} \backslash\{b\}\right)^{\bullet} \longleftarrow \operatorname{Tot}^{3} F\left(s, S^{3} \backslash\{b\}\right)^{\bullet} \longleftarrow \cdots \longleftarrow \operatorname{Tot}^{r} F\left(s, S^{3} \backslash\{b\}\right)^{\bullet} \longleftarrow \cdots
$$




\section{VOLIĆ}

where $\operatorname{Tot}^{r} F\left(s, S^{3} \backslash\{b\}\right)^{\bullet}$ is the homotopy fiber of the map

$$
\operatorname{Tot}^{r} X^{\bullet} \longrightarrow \operatorname{Tot}^{r} Y^{\bullet}=\Omega S^{2} .
$$

These homotopy fibers are exactly the partial totalizations of a cosimplicial space consisting of configuration spaces and doubling maps in fixed directions.

We then have a diagram

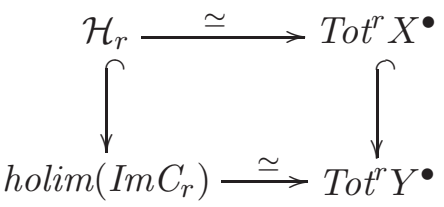

where the top equivalence is Theorem 5.6, and the bottom comes from the fact that both spaces are $\Omega S^{2}$ (see Proposition 5.12). It follows that the homotopy fibers of the two vertical inclusions are weakly equivalent. These fibers are precisely $T_{r}$ and $\operatorname{Tot}^{r} F\left(s, S^{3} \backslash\{b\}\right)^{\bullet}$.

Therefore, removing the spheres gives equivalent partial totalizations and homotopy limits of subcubical diagrams. From now on, we will refer to both as $T_{r}$ and which model we mean will be clear from the context. To simplify notation, we will continue to denote by $X^{\bullet}$ the new cosimplicial space differing from the old in that the tangential data has been removed.

\section{Finite type invariants and the Taylor tower}

Here we associate a spectral sequence to the tower (16) and deduce all the main results. This spectral sequence arises from any cosimplicial space and this is precisely the reason why we introduced $X^{\bullet}$. Its $E_{2}$ term will turn out to be the connection between the Taylor tower and finite type invariants.

\subsection{Weight systems and the cohomology spectral sequence}

This section briefly recalls the procedure for turning a cosimplicial space into a double complex from which the $E_{1}$ term of the cohomology spectral sequence is constructed. The spectral sequence then converges to the cohomology of the total complex of this double complex, which can be thought of as an algebraic analog of the totalization $\operatorname{Tot} X^{\bullet}$. In favorable cases, which may or may not be case here, the cohomology of the total complex will be isomorphic to the cohomology of the totalization of the cosimplicial space. Sinha [Sin02] shows this to be true for the Taylor tower for spaces of knots in $\mathbb{R}^{m}, m>3$, and we use this fact in [LV05] and [Vol04]. For more details about the construction of the spectral sequence for a cosimplicial space, see [Bou87, BK72].

Recall that $X^{\bullet}$ consists of spaces $X^{p}=F\left(p, S^{3} \backslash\{b\}\right), p \geqslant 0$ (tangent vectors have been removed). However, $S^{3} \backslash\{b\}$ is homeomorphic to $\mathbb{R}^{3}$. To simplify notation, we thus set

$$
F(p)=F\left(p, \mathbb{R}^{3}\right) \simeq F\left(p, S^{3} \backslash\{b\}\right) .
$$

We briefly recall the ring structure of the cohomology of $F(p)$. More details can be found in [Coh76].

Let $\nu$ denote the invariant unit volume form on $S^{2}$ as before. Let $\left(x_{1}, \ldots, x_{p}\right) \in F(p)$. Then we have maps

$$
x_{i j}: F(p) \longrightarrow S^{2}
$$

given by

$$
\left(x_{1}, \ldots, x_{p}\right) \longmapsto \frac{x_{j}-x_{i}}{\left|x_{j}-x_{i}\right|} .
$$

Each can be used to pull back $\nu$ to $F(p)$. Let $\alpha_{i j}=x_{i j}^{*} \nu$. Then $H^{q}(F(p))$ is 0 if $q$ is odd, and it is 


\section{Finite TYPE KNOT INVARIANTS AND THE CALCULUS OF FUNCTORS}

generated by products of $\alpha_{i j}$ with relations

$$
\begin{gathered}
\alpha_{i j}^{2}=0, \\
\alpha_{i j}=-\alpha_{j i}, \\
\alpha_{i j} \alpha_{k l}=\alpha_{k l} \alpha_{i j}, \\
\alpha_{i j} \alpha_{j k}+\alpha_{j k} \alpha_{k i}+\alpha_{k i} \alpha_{i j}=0,
\end{gathered}
$$

if $q$ is even.

The Poincaré polynomial for $F(p)$ is

$$
P_{p}(t)=\left(1+t^{2}\right)\left(1+2 t^{2}\right) \cdots\left(1+(p-1) t^{2}\right) .
$$

We can now set up a double cochain complex by applying cochains to the cosimplicial space:

$$
C^{*} X^{\bullet}=\left(C^{*} X^{1} \stackrel{\partial^{h}}{\longleftarrow} C^{*} X^{2} \stackrel{\partial^{h}}{\longleftarrow} C^{*} X^{3} \stackrel{\partial^{h}}{\longleftarrow} \cdots\right) .
$$

It is not hard to see that

$$
\partial^{h}=\left(\partial^{0}\right)^{*}-\left(\partial^{1}\right)^{*}+\left(\partial^{2}\right)^{*}-\cdots .
$$

gives a differential in the horizontal direction. Let the vertical differential be denoted by $\partial^{v}$.

Denoting the total complex of this double complex by $\operatorname{Tot} C^{*} X^{\bullet}$, there is a second quadrant spectral sequence converging to the cohomology of $\operatorname{Tot} C^{*} X^{\bullet}$. This cohomology has two filtrations coming from a choice of starting at $E_{1}$ by taking the cohomology with respect to $\partial^{h}$ or $\partial^{v}$. We use the latter and set up the spectral sequence so that

$$
E_{1}^{-p, q}=H^{q}(F(p)) .
$$

With (21) in hand, the $E_{1}$ term is easily computable. However, the double complex $C^{*} X^{\bullet}$ can in some way be 'simplified' prior to computing this page. Namely, instead of considering all of $C^{*} X^{\bullet}$, we only look at a certain subcomplex, $N C^{*} X^{\bullet}$, called the Dold-Kan normalization. It is constructed in the following way:

$$
\begin{gathered}
N C^{*} X^{0}=C^{*} X^{0} \\
N C^{*} X^{p}=C^{*} X^{p} / G^{p}, \quad p>0,
\end{gathered}
$$

where $G^{p}$ is the subgroup generated by

$$
\sum_{i=0}^{p-1} i m\left(C^{*} X^{p-1} \stackrel{\left(s^{i}\right)^{*}}{\longrightarrow} C^{*} X^{p}\right) .
$$

This normalization can be applied to any double complex obtained from a simplicial or a cosimplicial space. The important feature is that $\operatorname{Tot} N C^{*} X^{\bullet}$ has the same cohomology as $\operatorname{Tot} C^{*} X^{\bullet}$. The normalization, in general, can be thought of as throwing away the degenerate part of a (co)simplicial complex.

Let $G^{p, n}$ be the subgroup of $H^{2 n}(F(p))$ generated by the images of

$$
\left(s^{i}\right)^{*}: H^{2 n}(F(p-1)) \longrightarrow H^{2 n}(F(p)) .
$$

Then the normalized $E_{1}$ has entries

$$
E_{1}^{-p, 2 n}=H_{\text {norm }}^{2 n}(F(p))=H^{2 n}(F(p)) / G^{p, n} .
$$

Choose a basis for $H^{2 n}(F(p))$ using relations (17)-(20) by letting

$$
\alpha=\alpha_{i_{1} j_{1}} \alpha_{i_{2} j_{2}} \cdots \alpha_{i_{k} j_{k}}, \quad i_{m}, j_{m} \in\{1, \ldots, p\},
$$




\section{VOLIĆ}

be a generator of $H^{2 n}(F(p))$. Then $\alpha$ is a basis element if

$$
\begin{gathered}
i_{a}=i_{b} \Longrightarrow j_{a} \neq j_{b}, \\
i_{a}<j_{a}, \\
j_{1}<j_{2}<\cdots<j_{k},
\end{gathered}
$$

The first observation is as follows.

Lemma 6.1. If $2 n<p$, then $H_{\text {norm }}^{2 n}=0$.

Proof. Since $2 n<p$, it follows that some point $x_{r}$ in $F(p)$ was not used in any $x_{i j}$. Now apply the codegeneracy $s^{r}: F(p) \longrightarrow F(p-1)$ by forgetting $x_{r}$ and relabeling the result. Then $x_{i} \in F(p)$ remains $x_{i} \in F(p-1)$ if $i<r$, and becomes $x_{i-1} \in F(p-1)$ if $i>r$.

Now let $\alpha^{\prime}$ be a class in $H^{2 n}(F(p-1))$ constructed from the maps $x_{i j}^{\prime}$ such that

$$
x_{i j}^{\prime}= \begin{cases}x_{i(j-1)}, & \text { if } i<r<j, \\ x_{(i-1)(j-1)}, & \text { if } r<i<j, \\ x_{i j}, & \text { if } i<j<r .\end{cases}
$$

However, by construction, composing the product of the $x_{i j}^{\prime}$ with $s^{r}$ yields precisely $\alpha$. Thus, $\alpha$ is in the image of

$$
\left(s^{r}\right)^{*}: H^{2 n}(F(p-1)) \longrightarrow H^{2 n}(F(p)) .
$$

The discussion in the preceding proof can be extended to other cases. Regardless of the relation between $2 n$ and $p$, we can still conclude that if a class $\alpha$ is obtained without using all of the points in the configuration, some codegeneracy induces a map on cohomology with $\alpha$ in its image.

On the other hand, if $\alpha$ is obtained by using all of the points, then for every codegeneracy $s^{i}$, a map on $F(p)$ involving $x_{i}$ becomes undefined on $F(p-1)$. Thus, $\alpha$ is not in the image of $\left(s^{i}\right)^{*}$ for any $i$, nor is it in the subspace generated by them as the image of each codegeneracy is generated by a part of the basis. Hence, $\alpha$ survives to $H_{\text {norm }}^{2 n}(F(p))$. For the same reason, all such $\alpha$ remain independent after normalization. It follows therefore that a basis element $\alpha$ in $H_{\text {norm }}^{2 n}(F(p))$ can be described by simply adding one more requirement to conditions (25)-(27):

For every $x_{i} \in F(p), i$ must occur as a subscript in $\alpha$.

It is convenient at this stage to switch the point of view from cohomology to homology for establishing a clear connection between the Taylor tower (and its cosimplicial model) and finite type knot invariants.

Given a basis element $\alpha=\alpha_{i_{1} j_{1}} \alpha_{i_{2} j_{2}} \cdots \alpha_{i_{n} j_{n}} \in H_{\text {norm }}^{2 n}(F(p))$, let $a=a_{i_{1} j_{1} i_{2} j_{2} \cdots i_{n} j_{n}}$ denote its dual in $H_{2 n}^{\text {norm }}(F(p))$. (By $H_{2 n}^{\text {norm }}(F(p))$ we mean the homology obtained by considering chains on $X^{\bullet}$, using the alternating sums of $\left(\partial^{i}\right) *$ to obtain a chain complex in one direction, and then normalizing it via a dual version of (23).) An element $a \in H_{2 n}^{\text {norm }}(F(p))$ can be thought of as a chord diagram oriented by $p$ labeled points which are connected by oriented chords as prescribed by pairs of indices in $a$. Thus, $i_{1}$ is connected to $j_{1}$ and the arrow on the chord points from $i_{1} ; i_{2}$ is connected to $j_{2}$ with the arrow pointing from $i_{2}$, etc. We also impose the following sign convention: if two basis elements $a$ and $a^{\prime}$ differ by $\tau$ transpositions of their subscripts, then $a=(-1)^{\tau} a^{\prime}$. Thus, we choose any element $a$ and a sign for it, and then apply the sign convention. It is easy to see that this is a well-defined assignment.

Conversely, take a free module generated by chord diagrams with $p$ labeled vertices and $n$ oriented chords, and consider its quotient obtained by imposing the relations: 


\section{Finite TYPE KNOT INVARIANTS AND THE CALCULUS OF FUNCTORS}

(1) if a chord in a diagram $D$ connects the same vertex, then $D=0$;

(2) if two chords in $D$ connect the same vertices, then $D=0$;

(3) if two diagrams $D_{1}$ and $D_{2}$ differ by a change of orientation of a chord or by a transposition of two vertex labels, then $D=-D^{\prime}$;

(4) let $i<j<k$; if

$D_{1}$ contains two chords connecting vertices $i, j$ and $j, k$;

$D_{2}$ contains two chords connecting vertices $i, j$ and $i, k$;

$D_{3}$ contains two chords connecting vertices $i, k$ and $j, k$;

then $D_{3}=-D_{1}-D_{2}$.

It follows by construction that $H_{2 n}^{\text {norm }}(F(p))$ is isomorphic to this quotient. In the most relevant case for us, $p=2 n$, every vertex of a chord diagram must then have exactly one chord connecting it to some other vertex. However, this is precisely the description of $C D_{n}$. We thus have the following simple but important statement.

Proposition 6.2. $E_{-2 n, 2 n}^{1}=H_{2 n}^{\text {norm }}(F(2 n))$ is generated by $C D_{n}$.

The following proposition states that the first differential introduces precisely the $4 T$ relation of $\S 2$. We prove the dual version because the combinatorics of the proof will be more transparent. Remember that the space of weight systems $\mathcal{W}_{n}$ was defined as functions on $\mathbb{R}\left[C D_{n}\right]$ vanishing on the $4 T$ relation.

Proposition 6.3. $E_{2}^{-2 n, 2 n} \cong \mathcal{W}_{n}$.

Proof. Since $d^{1}=d^{h}$ and $H_{\text {norm }}^{2 n}(F(2 n+1))=0$, the image of $d^{1}$ in $H_{\text {norm }}^{2 n}(F(2 n))$ is 0 and so

$$
E_{2}^{-2 n, 2 n}=\operatorname{ker}\left(H_{\text {norm }}^{2 n}(F(2 n)) \stackrel{d^{1}}{\longrightarrow} H_{\text {norm }}^{2 n}(F(2 n-1))\right) .
$$

Let $\langle\cdot, \cdot\rangle$ denote evaluation of cohomology on homology, $f$ an element of $H_{\text {norm }}^{2 n}(F(2 n))$, and $B$ the basis of $H_{2 n}^{\text {norm }}(F(2 n-1))$. Then

$$
f \in \operatorname{ker}\left(d^{1}\right) \Longleftrightarrow\left\langle f, d_{1} a\right\rangle=0 \quad \forall a \in B,
$$

where $d_{1}$ is the alternating sum of maps induced by cofaces on homology.

Recall that $\partial^{r}$ sends $x_{i} \in F(2 n-1)$ to $x_{i} \in F(2 n)$ if $i \leqslant r$ and to $x_{i+1}$ if $i>r$. It follows that composing any map $x_{i j}: F(2 n) \longrightarrow S^{2}$ with $\partial^{r}$ gives a map

$$
x_{i j}^{\prime}: F(2 n-1) \longrightarrow S^{2},
$$

where $x_{i j}^{\prime}$ are given exactly as in (28), except $i=r$ and $j=r$ are also possibilities in the first and last case, respectively.

Then $\left(\partial^{r}\right)^{*}$ sends a basis element $\alpha$ to a basis element $\alpha^{\prime}$ by reindexing each two-dimensional class $\alpha_{i j}$ in the same way as in (28).

Note that $\alpha^{\prime}$ will have exactly one index that is repeated, as it should. Namely, indices $r$ and $r+1$ in $\alpha$ will both become $r$ in $\alpha^{\prime}$. However, any time $\alpha^{\prime}$ has as factors two classes $\alpha_{i r}^{\prime}$ and $\alpha_{j r}^{\prime}$, $i<j<r$, we may rewrite it in terms of two other basis elements using (20).

Now let

$$
\alpha^{\prime}=\alpha_{i_{1} j_{1}}^{\prime} \alpha_{i_{2} j_{2}}^{\prime} \cdots \alpha_{i_{n} j_{n}}^{\prime}
$$

be a basis element of $H_{\text {norm }}^{2 n}(F(2 n-1))$. Two of the indices must be the same either $\alpha^{\prime}$ contains $\alpha_{b c}^{\prime} \alpha_{b d}^{\prime}$ or $\alpha_{b c}^{\prime} \alpha_{c d}^{\prime}$ (the latter two 2 -forms do not necessarily have to be next to each other in $\alpha^{\prime}$ ). 


\section{VOLIĆ}

Consider the first case. There are four basis elements $\alpha \in H^{2 n}(F(2 n))$ which will yield this $\alpha^{\prime}$ under some $\left(\partial^{i}\right)^{*}$ :

$$
\begin{gathered}
\alpha_{1}=\alpha_{i_{1} j_{1}} \alpha_{i_{2} j_{2}} \cdots \alpha_{b(c+1)} \alpha_{(b+1)(d+1)} \cdots \alpha_{i_{n+1} j_{n+1}}, \\
\alpha_{2}=\alpha_{i_{1} j_{1}} \alpha_{i_{2} j_{2}} \cdots \alpha_{b(c+1)} \alpha_{(b+1)(d+1)} \cdots \alpha_{i_{n+1} j_{n+1}}, \\
\alpha_{3}=\alpha_{i_{1} j_{1}} \alpha_{i_{2} j_{2}} \cdots \alpha_{b d} \alpha_{c(d+1)} \cdots \alpha_{i_{n+1} j_{n+1}}, \\
\alpha_{4}=\alpha_{i_{1} j_{1}} \alpha_{i_{2} j_{2}} \cdots \alpha_{b d} \alpha_{c(d+1)} \cdots \alpha_{i_{n+1} j_{n+1}} .
\end{gathered}
$$

Here, $\alpha_{1}$ and $\alpha_{2}$ give exactly $\alpha^{\prime}$ under $\left(\partial^{b}\right)^{*}$. However, $\left(\partial^{d}\right)^{*}$, applied to $\alpha_{3}$ and $\alpha_{4}$, produces an element which is identical to $\alpha^{\prime}$ except for the factor $\alpha_{b c}^{\prime} \alpha_{b d}^{\prime}$. This factor is, in fact, replaced by $\alpha_{b d}^{\prime} \alpha_{c d}^{\prime}$. However, this is not an element in the basis of $H_{\text {norm }}^{2 n}(F(2 n-1))$, and it can be rewritten as $-\alpha^{\prime}-\alpha^{\prime \prime}$, where $\alpha^{\prime \prime}$ contains $\alpha_{b c}^{\prime} \alpha_{c d}^{\prime}$. Note that the four forms $\alpha_{i}$ only differ in the two factors as indicated.

Similarly, in the second case of a possible repeated index in $\alpha^{\prime}$, we again get four basis elements $\alpha_{i}$ with $\alpha_{3}$ and $\alpha_{4}$ the same as above, and with

$$
\begin{aligned}
& \alpha_{1}=\alpha_{i_{1} j_{1}} \alpha_{i_{2} j_{2}} \cdots \alpha_{b(c+1)} \cdots \alpha_{c(d+1)} \cdots \alpha_{i_{n+1} j_{n+1}}, \\
& \alpha_{2}=\alpha_{i_{1} j_{1}} \alpha_{i_{2} j_{2}} \cdots \alpha_{b c} \cdots \alpha_{(c+1)(d+1)} \cdots \alpha_{i_{n+1} j_{n+1}},
\end{aligned}
$$

giving $\alpha^{\prime}$ via $\left(\partial^{c}\right)^{*}$.

Let $a^{\prime}, a_{i} \in H_{2 n}^{\text {norm }}(F(2 n))$ be dual to the elements $\alpha^{\prime}, \alpha_{i}$. We have thus shown that there are exactly four elements $\alpha \in H_{\text {norm }}^{2 n}(F(2 n))$ whose $d^{1}$, evaluated on $a^{\prime}$ is nonzero. More precisely,

$$
\left\langle d^{1} \alpha_{1}, a^{\prime}\right\rangle=1, \quad\left\langle d^{1} \alpha_{2}, a^{\prime}\right\rangle=-1, \quad\left\langle d^{1} \alpha_{3}, a^{\prime}\right\rangle=-1, \quad\left\langle d^{1} \alpha_{4}, a^{\prime}\right\rangle=1 .
$$

The signs depend on which index in $a^{\prime}$ is repeated (as $d^{1}$ is an alternating sum), as well as indices $b, c$, and $d$ (the number of transpositions needed to get from one labeling to another has to be taken into account). So

$$
d_{1} a^{\prime}=a_{1}-a_{2}-a_{3}+a_{4}
$$

and we conclude that

$$
f \in \operatorname{ker}\left(d^{1}\right) \Longleftrightarrow\left\langle f, a_{1}-a_{2}-a_{3}+a_{4}\right\rangle=0
$$

for all $a_{1}, a_{2}, a_{3}$, and $a_{4}$ whose indices are related as in (30) or (31).

If we recall that classes $a \in H_{2 n}^{\text {norm }}(F(2 n))$ were associated to chord diagrams with $n$ chords, then the conditions on $a_{i}$ precisely describe the four chord diagrams in the $4 T$ relation. Thus, we get that, if $f$ is thought of as a function on $C D_{n}$, then

$$
f \in E_{2}^{-2 n, 2 n} \Longleftrightarrow\langle f, 4 T\rangle=0 \text { for all } 4 T \text { relations in } C D_{n} \Longleftrightarrow f \in \mathcal{W}_{n} .
$$

\subsection{The cotower of partial totalizations}

Recall that, in the normalized $E_{2}$ term of the spectral sequence, we have

$$
E_{2}^{-p, q}=0 \text { if } p<q \text { or } p=q=2 k+1 \text { for some } k .
$$

Since

$$
E_{i+1}^{-p, q}=\operatorname{ker}\left(E_{i}^{-p, q} \stackrel{d^{i}}{\longrightarrow} E_{i}^{-p+i, q-i+1}\right) / i m\left(E_{i}^{-p-i, q+i-1} \stackrel{d^{i}}{\longrightarrow} E_{i}^{-p, q}\right),
$$

we have, for $p=q$,

$$
E_{i+1}^{-p, p}=\operatorname{ker}\left(E_{i}^{-p, p} \stackrel{d^{i}}{\longrightarrow} E_{i}^{-p+i, p-i+1}\right) .
$$

An immediate consequence is that we can construct an algebraic analog of $T_{r}$, called $T_{r}^{*}$, as follows. We truncate $X^{\bullet}$ at the $r$ th space for any $r$, and then construct the double cochain complex (now finite in the horizontal direction) and the associated spectral sequence. Letting $T_{r}^{*}$ be the total 


\section{Finite TYPE KNOT INVARIANTS AND THE CALCULUS OF FUNCTORS}

complex of this double complex, the spectral sequence would now be computing the cohomology of $T_{r}^{*}$. We then have

$$
H^{0}\left(T_{r}^{*}\right)=\bigoplus_{j=0}^{r} E_{\infty}^{-j, j} .
$$

Now consider $H^{0}\left(T_{r+1}^{*}\right)$. A consequence of $(33)$ is that its grading would be exactly the same as that of $H^{0}\left(T_{r}^{*}\right)$, but with one more summand. In short, there would be no new differentials coming into the diagonal of the spectral sequence in passing from the truncation of $X^{\bullet}$ at $r$ to $r+1$. Since every odd entry on the diagonal at the $E_{2}$ term is 0 , we conclude that

$$
\begin{gathered}
H^{0}\left(T_{2 n}^{*}\right) \cong H^{0}\left(T_{2 n+1}^{*}\right) \\
H^{0}\left(T_{2 n}^{*}\right) / H^{0}\left(T_{2 n-1}^{*}\right) \cong E_{\infty}^{-2 n, 2 n} .
\end{gathered}
$$

We can put the above isomorphisms (the second giving an injection from $H^{0}\left(T_{2 n-1}^{*}\right)$ to $H^{0}\left(T_{2 n}^{*}\right)$ ) together into a tower of invariants of partial totalizations which we call a Taylor cotower:

$$
\cdots \stackrel{\cong}{\longrightarrow} H^{0}\left(T_{2 n-1}^{*}\right) \longleftrightarrow H^{0}\left(T_{2 n}^{*}\right) \stackrel{\cong}{\longrightarrow} H^{0}\left(T_{2 n+1}^{*}\right) \longleftrightarrow \cdots
$$

Each space comes with a map $e v_{[i]}^{*}$ to $H^{0}(\overline{\mathcal{K}})$ described below.

Remark. Going from partial totalizations $T_{r}=\operatorname{Tot}^{r} X^{\bullet}$ to total complexes $T_{r}^{*}=\operatorname{Tot}^{r} C^{*} X^{\bullet}$ is also possible directly from the subcubical diagrams of configuration spaces. We have bypassed this by working with $X^{\bullet}$ instead, but we could have also considered cochains on all spaces of punctured embeddings in the subcubical diagram (all arrows would now get reversed) and then formed the homotopy colimit hocolim $\left(C^{*} F C_{r}\right)$ (dual to the homotopy limit, but now in an algebraic sense). A double complex $T_{r}^{*}$ could then be formed by collecting all cochains on embeddings with the same number of punctures.

\subsection{The evaluation map to finite type invariants}

Let $e v_{[i]}^{*}$ be the map induced by $e v_{[i]}$ on cochains on configurations and cohomology:

$$
e v_{[i]}^{*}: H^{0}\left(\operatorname{Tot}^{i} C^{*} X^{\bullet}\right)=H^{0}\left(T_{i}^{*}\right) \longrightarrow H^{0}(\overline{\mathcal{K}}) .
$$

To simplify notation, we set $e v^{*}=e v_{[i]}^{*}$ for all $i$. Which value of $i$ is used will be clear from the context throughout the section.

Now remember from the previous section that $H^{0}\left(T_{2 n}^{*}\right) / H^{0}\left(T_{2 n-1}^{*}\right)=E_{\infty}^{-2 n, 2 n}$.

Proposition 6.4. The image of $e v^{*}$ is contained in $\mathcal{V}_{n} / \mathcal{V}_{n-1}$. Further, the diagram

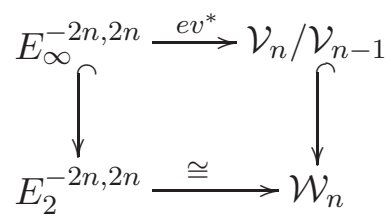

commutes.

For clarity, it is better to prove this proposition using the tower which is dual to that in (36). As mentioned in $\S 6.1$, we could have started with chains on the cosimplicial space $X^{\bullet}$ and formed the totalizations $T_{*}^{i}=\operatorname{Tot}^{i} C_{*} X^{\bullet}$, where $\operatorname{Tot}^{i} C_{*} X^{\bullet}$ now means the total complex of a double chain complex. The tower of totalizations then on homology looks like

$$
\cdots \lll H_{0}\left(T_{*}^{2 n-1}\right) \longleftarrow H_{0}\left(T_{*}^{2 n}\right) \longleftarrow H_{0}\left(T_{*}^{2 n+1}\right) \ll-\cdots .
$$




\section{VOLIĆ}

There are also maps $e v_{*}$ from $H_{0}(\overline{\mathcal{K}})$ to each stage, induced by $e v_{[i]}$ for each $i$ on chains and homology. Since we are ultimately interested in detecting finite type invariants in the cotower and, consequently, in mapping sums of resolutions of singular knots into the stages of the homology tower, we introduce an equivalent description of this tower.

Given a space $X$, one can consider the free abelian group $\mathbb{R} X$ of linear combinations of points in $X$. This is a space that inherits its topology from $X$ and is related to the infinite symmetric product of $X$. The most important characterization of this space is a version of the Dold-Thom theorem, whose proof can be found in [DT58, Hat01].

TheOREM 6.5. The homotopy groups of $\mathbb{R} X$ are isomorphic to the homology groups of $X$ with real coefficients.

The tower we will then use to prove Proposition 6.4 is the following.

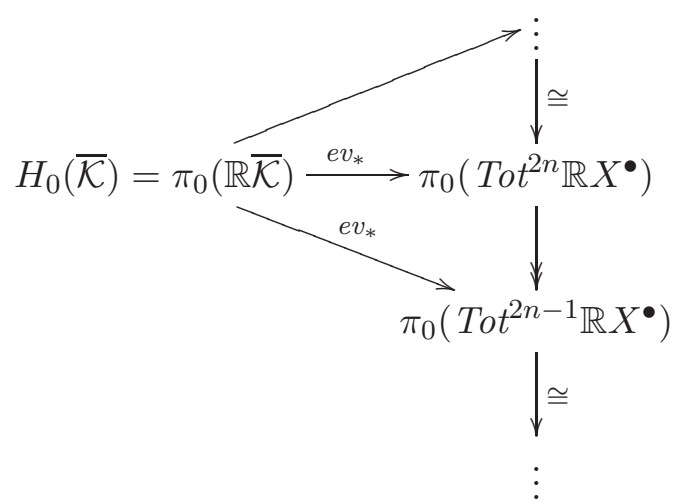

The advantage of this tower is that a singular knot can now be mapped into it by a map of the linear combination of its resolutions (with appropriate signs as dictated by the Vassiliev skein relation).

Lemma 6.6. The towers (37) and (38) are equivalent.

Proof. Let:

- $\mathcal{C}$ be the category of chain complexes;

- $\mathcal{S}$ the category of spectra; and

- $\mathcal{T}$ the category of topological spaces.

Given $C_{*} \in \mathcal{C}$, define $T^{i} C_{*}$ to be the truncation of $C_{*}$,

$$
T^{i} C_{*}=\operatorname{ker}(\partial) \stackrel{\partial}{\longleftarrow} C_{i+1} \stackrel{\partial}{\longleftarrow} C_{i+2} \stackrel{\partial}{\longleftarrow} \cdots .
$$

A simplicial abelian group $A^{i}$ can be associated to each $T^{i} C_{*}$ by the Dold-Kan construction [Dol58, Kan58] (in fact, $\mathcal{C}$ and $\mathcal{A}$ are equivalent; see also [May67]). The realization functor $|\cdot|$ can then be applied to $A^{i}$ to yield a space we denote by $\left|T^{i} C_{*}\right|$. Further, one can then pass to $\mathcal{S}$ by collecting these spaces as

$$
\left\{\left|T^{0} C_{*}\right|,\left|\Sigma T^{-1} C_{*}\right|,\left|\Sigma^{2} T^{-2} C_{*}\right|, \ldots\right\} .
$$

Here $\Sigma$ means an algebraic suspension which shifts the truncation of $C_{*}$, so that the above spectrum is, in fact, an $\Omega$-spectrum. We denote the resulting composed functor from $\mathcal{C}$ to $\mathcal{S}$ by $F$.

The homotopy of $\left|T^{0} C_{*}\right|$ can be shown to be the same as the (non-negative) homology of $C_{*}$. On the other hand, we can apply the functor $\Omega^{\infty}$ to $F\left(C_{*}\right)$ and get a space whose homotopy is the homotopy of $\left|T^{0} C_{*}\right|$ since $F\left(C_{*}\right)$ is an $\Omega$-spectrum. 


\section{Finite TYPE KNOT INVARIANTS AND THE CALCULUS OF FUNCTORS}

Now, given a space $X$, we can do the same. Consider the composition

$$
\begin{gathered}
\mathcal{T} \longrightarrow \mathcal{C} \longrightarrow \mathcal{S} \longrightarrow \mathcal{T} \\
X \longmapsto C_{*} X \longmapsto F\left(C_{*} X\right) \longmapsto \Omega^{\infty} F\left(C_{*} X\right) \sim\left|T^{0} C_{*} X\right| \sim \mathbb{R} X .
\end{gathered}
$$

The first equivalence in $\mathcal{T}$ is again due to the fact that $F\left(C_{*} X\right)$ is an $\Omega$-spectrum. The second is given by Theorem 6.5 since the homotopy of $F\left(C_{*} X\right)$ is the homology of $\left|T^{0} C_{*} X\right|$, which, in turn, is the homology of $X$.

However, we can go even further, and start with a cosimplicial space $X^{\bullet}$ (or any diagram of spaces viewed as a functor from an indexing category to $\mathcal{T}$ ). A generalized version of the above produces a cosimplicial spectrum $F\left(C_{*} X^{\bullet}\right)$ and we get a composition of functors

$$
X^{\bullet} \longmapsto C_{*} X^{\bullet} \longmapsto F\left(C_{*} X^{\bullet}\right) \longmapsto \Omega^{\infty} F\left(C_{*} X^{\bullet}\right) \sim \mathbb{R} X^{\bullet} .
$$

In particular, from the weak equivalence in the above, we can deduce

$$
\operatorname{Tot}^{r}\left(\Omega^{\infty} F\left(C_{*} X^{\bullet}\right)\right) \sim \operatorname{Tot}^{r}\left(\mathbb{R} X^{\bullet}\right),
$$

for all partial totalizations $\operatorname{Tot}^{r}$.

Remarks. Totalization only preserves equivalences, however, if $X^{\bullet}$ is a fibrant cosimplicial space. Every cosimplicial space can be replaced by a fibrant one via a degreewise equivalence of cosimplicial spaces and, in fact, our cosimplicial space had to be replaced by a fibrant one for Theorem 5.6 to be true anyway.

For a more general diagram of spaces, the role of the totalization is played by the homotopy limit of the diagram.

Next we have

$$
\Omega^{\infty} F\left(\operatorname{Tot}^{r} C_{*} X^{\bullet}\right) \sim \Omega^{\infty} \operatorname{Tot}^{r}\left(F\left(C_{*} X^{\bullet}\right)\right) \sim \operatorname{Tot}^{r}\left(\Omega^{\infty} F\left(C_{*} X^{\bullet}\right)\right) .
$$

The second equivalence is true because the functor $\operatorname{Tot}^{r}$ is defined as a compatible collection of spaces $\operatorname{Maps}\left(\Delta^{i}, X^{i}\right), i \leqslant r$, and $\Omega \operatorname{Maps}\left(\Delta^{i}, X^{i}\right) \sim \operatorname{Maps}\left(\Delta^{i}, \Omega X^{i}\right)$. Essentially the same reasoning applies in the case of the first equivalence, except the argument is not as straightforward since $\operatorname{Tot}^{r} C_{*} X^{\bullet}$ is an algebraic construction involving total complexes rather than mapping spaces.

However, we also know that there is an equivalence

$$
\Omega^{\infty} F\left(\operatorname{Tot}^{r} C_{*} X^{\bullet}\right) \sim \operatorname{Tot}^{r} \mathbb{R} X .
$$

In particular, putting (39), (40), and (41) together on $\pi_{0}$, we have

$$
\pi_{0}\left(\operatorname{Tot}^{r} \mathbb{R} X^{\bullet}\right)=H_{0}\left(\operatorname{Tot}^{r} C_{*} X^{\bullet}\right) .
$$

Since the tower (37) had $H_{0}\left(\operatorname{Tot}^{r} C_{*} X^{\bullet}\right)=H_{0}\left(T_{*}^{r}\right)$ as its stages, we can now replace them by $\pi_{0}\left(\operatorname{Tot}^{r} \mathbb{R} X^{\bullet}\right)$.

Proof of Proposition 6.4. The idea will be that an $n$-singular knot maps to $\operatorname{Tot}^{2 n-1} \mathbb{R} X^{\bullet}$ via the evaluation map, but the map is homotopic to 0. However, the homotopy cannot be extended to $\operatorname{Tot}^{2 n} \mathbb{R} X^{\bullet}$ and the obstruction to doing so is precisely the chord diagram associated to the singular knot.

So let $K$ be an $n$-singular knot, and let $\left\{a_{1}, \ldots, a_{n}, b_{1}, \ldots, b_{n}\right\}$ be the points in the interior of $I=[0,1]$ making up the singularities, i.e. $K\left(a_{i}\right)=K\left(b_{i}\right)$. Let $t=\left(t_{1}, \ldots, t_{j}\right)$ be a point in $\Delta^{j}$ and note that for all $j$ we are considering, $j<2 n$. The fact that there are always fewer $t_{i}$ than $a_{i}$ and $b_{i}$ is crucial in what follows. 


\section{VOLIĆ}

Now pick closed intervals $I_{k}, J_{k}$ around the points $a_{k}, b_{k}$ so that the $2^{n}$ resolutions of $K$ differ only in the $K\left(I_{k}\right)$. Let the resolutions be indexed by the subsets $S$ of $\{1,2, \ldots, n\}$ in the sense that if $k \in S$, then $K\left(I_{k}\right)$ was changed into an overstrand (in the Vassiliev skein relation).

It is clear how to extend $e v$ to linear combinations of knots and get a map

$$
e v: \mathbb{R} \overline{\mathcal{K}} \longrightarrow\left(\Delta^{j} \rightarrow \mathbb{R} X^{j}\right) .
$$

In particular, we can map sums of resolutions $K_{S}$ (taken with appropriate signs) of an $n$-singular knot.

Let

$$
\begin{array}{ll}
e_{k}^{+}=K_{S}\left(I_{k}\right), & \text { if } k \in S, \\
e_{k}^{-}=K_{S}\left(I_{k}\right), & \text { if } k \notin S,
\end{array}
$$

and define a homotopy (parametrized by $u$ )

$$
e_{k}^{u}=(1-u) e_{k}^{+}+u e_{k}^{-}
$$

Note that this homotopy is only defined on $I_{k}$ and has the effect of changing an overcrossing to an undercrossing. The problem is that, for some time $u$, this homotopy reintroduces the original singularity on the resolved knot. This means that $e v$ will yield a degenerate configuration if any two of the $t_{i}$ equal $a_{k}$ and $b_{k}$.

To avoid this, it suffices to modify the homotopy so that no movement takes place when there is some $t_{i} \in \Delta^{j}$ which equals $b_{k}$. To do this, define a map

$$
\phi_{k}(t): \Delta^{j} \longrightarrow[0,1]
$$

by

$$
\phi_{k}(t)= \begin{cases}\frac{2 \min _{l}\left(\left|t_{l}-b_{k}\right|\right)}{\left|J_{k}\right|}, & \text { if at least one of the } t_{i} \text { is in } J_{k}, \\ 1, & \text { otherwise. }\end{cases}
$$

Then $\phi_{k}(t)$ is a continuous map which is 0 if, for any $i, t_{i}=b_{k}$ and 1 if, for all $i, t_{i} \notin J_{k}$.

Now let

$$
e_{S}^{u, t}= \begin{cases}e_{k}^{u \phi_{k}(t)}, & \text { in } I_{k} \text { if } k \in S, \\ K_{S}, & \text { outside } I_{k} \text { for all } k .\end{cases}
$$

Thus, $e_{S}^{u, t}$ changes overcrossing $K_{S}\left(I_{k}\right)$ to undercrossing $K_{S}\left(J_{k}\right)$ if no $t_{i}$ is in $J_{k}$. If some $t_{i}$ enters $J_{k}$, the homotopy happens only part of the way. If any $t_{i}$ comes to the center of $J_{k}$ and thus equals $b_{k}$, the homotopy is just constant.

This setup then prevents the collision of configuration points in $X^{j}$ so that it makes sense to use $e v$ to define a homotopy

$$
K_{S} \times I \longrightarrow\left(\Delta^{j} \rightarrow X^{j}\right)
$$

by

$$
\left(K_{S}, u\right) \mapsto e v\left(e_{S}^{u, t}\right)(t) .
$$

Adding over all $S \subset\{1, \ldots, n\}$ with appropriate signs, we get a homotopy

$$
\sum_{S}( \pm)\left(K_{S} \times I\right) \longrightarrow\left(\Delta^{j} \rightarrow \mathbb{R} X^{j}\right)
$$

We wish for this homotopy to be 0 when $u=1$. It is important to keep in mind that, for every $K_{S}$ and every $k$, there exists a $K_{S^{\prime}}$ such that $e v\left(K_{S}\right)$ and $e v\left(K_{S^{\prime}}\right)$ differ only in those $t_{i}$ which are in $I_{k}$, and these two configurations have opposite signs. 


\section{Finite TYPE KNOT INVARIANTS AND THE CALCULUS OF FUNCTORS}

We pick a point $t \in \Delta^{j}$ and distinguish the following cases.

Case 1. For all $i$ and all $k, t_{i} \notin I_{k}$.

Then $e v\left(e_{S}^{u, t}\right)(t)$ is the same configuration for all $S$ and for all $u$, and these cancel out in $\mathbb{R} X^{j}$. Note that this happens regardless of whether $J_{k}$ contains any of the $t_{i}$ or not.

Case 2. There exist $i$ and $k$, such that $t_{i} \in I_{k}$.

Case 2a. For all $j, t_{j} \notin J_{k}$.

Since $J_{k}$ is free of the $t_{j}$, ev $\left(e_{S}^{u, t}\right)(t)$ moves the overstrand $K_{S}\left(I_{k}\right)$ to the understrand $K_{S}\left(I_{k}\right)$ without introducing a singularity. So now the two resolutions are the same and they cancel because of the difference in signs. Since all the resolutions can be combined in pairs which only differ in one crossing, everything adds up to 0 .

Case 2b. There exist $j$ such that $t_{j} \in J_{k}$.

In this case, the homotopy $e v\left(e_{S}^{u, t}\right)(t)$ does not change the overcrossing to the undercrossing (it might perform a part of the movement, depending on where exactly $t_{j}$ is in $J_{k}$-it is important here that this partial movement happens on half of the resolutions which will pair up and cancel, as will the other half). However, since $j<2 n$, there must be an interval, either $I_{k^{\prime}}$ or $J_{k^{\prime}}$, which is free of all the $t_{l}$. This puts us back into one of the previous cases, with $k=k^{\prime}$. Everything cancels in pairs again.

This exhausts all of the cases so that

$$
\sum_{S}( \pm) K_{S} \longrightarrow\left(\Delta^{j} \rightarrow \mathbb{R} X^{j}\right)
$$

is homotopic to 0 for $1 \leqslant j \leqslant 2 n-1$. In other words, the map

$$
e v_{*}: \pi_{0}(\mathbb{Z} E) \longrightarrow \pi_{0}\left(\Delta^{j} \rightarrow \mathbb{R} X^{j}\right)
$$

is 0 on resolutions of $n$-singular knots for $1 \leqslant j \leqslant 2 n-1$.

We still need this to be a homotopy in $T o t^{2 n-1} \mathbb{R} X^{\bullet}$, so that we need to check the compatibility with coface and codegeneracy maps in $X^{\bullet}$. This, however, is immediate from the definition of $\phi_{k}(t)$ and we omit the details. Therefore,

$$
e v_{*}: \pi_{0}(\mathbb{Z} E) \longrightarrow \pi_{0}\left(T o t^{2 n-1} \mathbb{Z} E\right)
$$

is 0 on $n$-singular knots.

If we try to apply the same construction to $\operatorname{Tot}^{2 n} \mathbb{R} X^{\bullet}$, we will get 0 at $u=1$ if $j<2 n$, but Case $2 \mathrm{~b}$ now breaks down when $j=2 n$. This is because the $t_{i}$ could land in all $I_{k}$ and $J_{k}$. Part of the homotopy might still take place (depending on exactly where the $t_{i}$ are in those intervals), but there will be no cancellations among the configurations. We distinguish an extreme case which will be needed later, namely let

$$
t^{*}=\left\{a_{1}, \ldots, a_{n}, b_{1}, \ldots, b_{n}\right\} \in \Delta^{2 n} .
$$

However, since $e v\left(e_{S}^{u, t}\right)(t)$ still produces 0 outside of

$$
B^{2 n}=I_{1} \times J_{1} \times \cdots \times I_{n} \times J_{n} \in \operatorname{int}\left(\Delta^{2 n}\right),
$$

we have, at $u=1$, a map $\Delta^{2 n} \rightarrow \mathbb{R} X^{2 n}$ which takes everything outside of $B^{2 n}$ to 0 . In particular, the boundary of $\Delta^{2 n}$ goes to 0 , so we in fact have a map $S^{2 n} \rightarrow \mathbb{R} X^{2 n}$, or an element of $\pi_{2 n}\left(\mathbb{R} X^{2 n}\right) \cong$ $H_{2 n}\left(X^{2 n}\right)$. To see which element this is, we turn to cohomology.

Since $X^{2 n}$ is the configuration space $F\left(2 n, \mathbb{R}^{3}\right)$, we in fact have a map $B^{2 n} \rightarrow \mathbb{R} F\left(2 n, \mathbb{R}^{3}\right)$ and various maps $\mathbb{R} F\left(2 n, \mathbb{R}^{3}\right) \rightarrow \mathbb{R} S^{2}$ given by the normalized difference of two points in the configuration. Since we are looking for an element of $H^{2 n}$, we take $n$ such maps to pull back a $2 n$-form from a product of $n$ spheres. 


\section{VOLIĆ}

Now remember that $B^{2 n}$ is a product of $2 n$ disjoint intervals, so that the composition

$$
B^{2 n} \rightarrow \mathbb{R} F\left(2 n, \mathbb{R}^{3}\right) \rightarrow\left(\mathbb{R} S^{2}\right)^{n}
$$

breaks up as $n$ maps

$$
I_{k} \times J_{k^{\prime}} \rightarrow S^{2} \quad\left(\text { or } I_{k} \times I_{k^{\prime}} \text { or } J_{k} \times J_{k^{\prime}}\right) .
$$

However, the boundary of each of these squares maps to 0 , so we actually have $n$ maps from $S^{2}$ to $S^{2}$. Every such map is determined up to homotopy by its degree. We will therefore first pick a point $\left(v_{1}, \ldots, v_{n}\right)$ in $\left(S^{2}\right)^{n}$ which is a regular value for the composition

$$
B^{2 n} \rightarrow \mathbb{R} F\left(2 n, \mathbb{R}^{3}\right) \rightarrow\left(\mathbb{R} S^{2}\right)^{n} .
$$

However, since the linear combination of knots is here given by the resolutions of an $n$-singular knot, we may pick a point which is a regular value for

$$
B^{2 n} \rightarrow F\left(2 n, \mathbb{R}^{3}\right) \rightarrow\left(S^{2}\right)^{n}
$$

for all resolutions $S$. Then we will count the number of preimages of this regular value over all $S$ and all possible vectors between points in the configuration lying in the images of intervals $I_{k}$ and $J_{k}$. This will give an element of $H^{2 n}\left(X^{2 n}\right)$ whose dual is precisely the element which prevents the extension of the homotopy to $T o t^{2 n} \mathbb{R} X^{\bullet}$.

Let $S=\{1, \ldots, n\}$ and choose $\left(v_{1}^{*}, \ldots, v_{n}^{*}\right) \in\left(S^{2}\right)^{n}$ such that $v_{i}^{*}$ is the (normalized) vector which points from $e v\left(K_{S}\right)\left(b_{i}\right)$ to $e v\left(K_{S}\right)\left(a_{i}\right)$. Then the only time the map $F\left(2 n, \mathbb{R}^{3}\right) \rightarrow\left(S^{2}\right)^{n}$ produces this value is when $t=t^{*}$. Also, this value occurs only once over all the resolutions, and that is when the singular knot is resolved positively at all the singularities (so $K\left(I_{k}\right)$ is always the overstrand). Other simple geometric considerations show that this is the only preimage.

We may thus think of the cohomology class we obtain as represented by $n$ vectors pointing from the images of the $a_{i}$ to the images of the $b_{i}$. However, then the dual class may be represented by a chord diagram which is precisely the diagram associated to the $n$-singular knot we started with.

Finally note that $e v_{*}: \pi_{0}(\mathbb{R} \overline{\mathcal{K}}) \rightarrow \pi_{0}\left(T o t^{2 n-1} \mathbb{R} X^{\bullet}\right)$ is 0 for any singular knot with $n$ or more singularities, but it is not 0 on knots with $n-1$ singularities (repeat the arguments with a shift - the key is that we now would not have $j<2(n-1)$ for all $j)$.

We can now collect all of the information and prove the proposition as stated. Let

$$
\begin{gathered}
\overline{\mathcal{K}}_{n}=\{\text { sums of resolutions of } n \text {-singular knots }\} \\
{\left[\mathbb{R} \overline{\mathcal{K}}_{n}\right]=\left\{\text { homotopy classes of combinations of elements of } \overline{\mathcal{K}}_{n}\right\} .}
\end{gathered}
$$

Note that there is a filtration

$$
\pi_{0}(\mathbb{R} \overline{\mathcal{K}}) \supset\left[\mathbb{R} \overline{\mathcal{K}}_{1}\right] \supset \cdots \supset\left[\mathbb{R} \overline{\mathcal{K}}_{n}\right] \supset\left[\mathbb{R} \overline{\mathcal{K}}_{n+1}\right] \supset \cdots
$$

since every $(n+1)$-singular knot resolves into two $n$-singular knots. Also, observe that

$$
\mathcal{V}_{n}=\left(\pi_{0}(\overline{\mathcal{K}}) /\left[\mathbb{R} \overline{\mathcal{K}}_{n+1}\right]\right)^{*}
$$

and so

$$
\mathcal{V}_{n} / \mathcal{V}_{n-1}=\left(\left[\mathbb{R} \overline{\mathcal{K}}_{n}\right] /\left[\mathbb{R} \overline{\mathcal{K}}_{n+1}\right]\right)^{*}
$$

We have thus produced a map

$$
H_{0}(\overline{\mathcal{K}})=\pi_{0}(\mathbb{R} \overline{\mathcal{K}}) \supset\left[\mathbb{R} \overline{\mathcal{K}}_{n}\right] \longrightarrow \operatorname{ker}\left(\pi_{0}\left(T o t^{2 n} \mathbb{R} X^{\bullet}\right) \rightarrow \pi_{0}\left(T o t^{2 n-1} \mathbb{R} X^{\bullet}\right)\right) .
$$

Dual to the statement from the previous section that the quotient on cohomology is $E_{\infty}^{-2 n, 2 n}$, the above kernel is precisely $E_{-2 n, 2 n}^{\infty}$. On the other hand, this is a subgroup of $E_{-2 n, 2 n}^{2}$ which Proposition 6.3 shows (dually) to be isomorphic to $\mathcal{D}_{n}^{c}$. Thus, we have a map

$$
\left[\mathbb{R} \overline{\mathcal{K}}_{n}\right] \longrightarrow E_{-2 n, 2 n}^{\infty} \subseteq E_{-2 n, 2 n}^{2} \cong \mathcal{D}_{n}^{c}
$$




\section{Finite TYPE KNOT INVARIANTS AND THE CALCULUS OF FUNCTORS}

The fact that our homotopy produces 0 in $\pi_{0}\left(\operatorname{Tot}^{2 n} \mathbb{R} X^{\bullet}\right)$ on singular knots with more than $n$ singularities means that (45) actually maps from the combinations of resolutions of $n$-singular knots which are not combinations of resolutions of $(n+1)$-singular knots, so that we may write

$$
e v_{*}:\left[\mathbb{R} \overline{\mathcal{K}}_{n}\right] /\left[\mathbb{R} \overline{\mathcal{K}}_{n+1}\right] \longrightarrow E_{-2 n, 2 n}^{\infty} .
$$

Using (44), the dual of this map is precisely that in the statement of the proposition:

$$
e v^{*}: E_{\infty}^{-2 n, 2 n} \longrightarrow \mathcal{V}_{n} / \mathcal{V}_{n-1} \text {. }
$$

For the second part, recall that the chord diagram we obtained in $E_{-2 n, 2 n}^{2}$ was the same one that produced the singular knot in the usual way. It follows immediately that we have the following commutative diagram

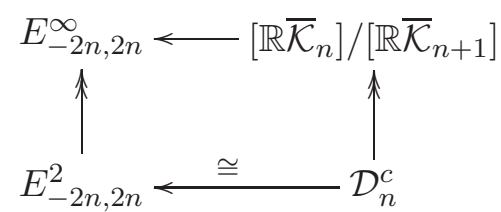

The surjection on the right is simply the statement that every $n$-singular knot can be obtained from a chord diagram by embedding an interval while identifying the points paired off by chords. The dual diagram is then

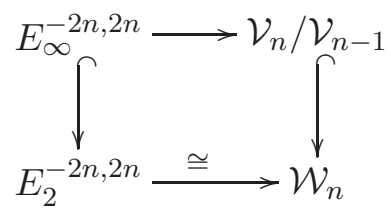

as desired.

The first part of Proposition 6.4 thus essentially states that any knot invariant which factors through the tower has to be finite type. In particular, recall Theorem 4.2 and note that $T(W)$ can be extended linearly to sums of resolutions of singular knots, thereby fitting the composition

$$
\left[\mathbb{R} \overline{\mathcal{K}}_{n}\right] \stackrel{e v_{*}}{\longrightarrow} \pi_{0}\left(T o t^{2 n} \mathbb{R} X^{\bullet}\right)=H_{0}\left(T_{*}^{2 n}\right) \stackrel{T(W)}{\longrightarrow} \mathbb{R} .
$$

However, we now know that this composition must vanish on $\left[\mathbb{R} \overline{\mathcal{K}}_{n+1}\right]$, so that we immediately have the following.

Corollary 6.7. The invariant $T(W): \pi_{0}\left(\mathcal{H}_{2 n}\right) \longrightarrow \mathbb{R}$, when extended to

$$
\pi_{0}\left(\operatorname{holim}\left(\mathbb{R} E C_{2 n}\right)\right)=\pi_{0}\left(\operatorname{Tot}^{2 n} \mathbb{R} X^{\bullet}\right)=H_{0}\left(T_{*}^{2 n}\right),
$$

vanishes on points in $T_{*}^{2 n}$ which come from sums of resolutions of $(n+1)$-singular knots. Thus $T(W)$ is a type $n$ invariant.

\subsection{Proofs of the main theorems}

We finally prove Theorems 1.2 and 1.3, thus in particular showing that the algebraic analog (36) of the Taylor tower for $\overline{\mathcal{K}}$ classifies finite type invariants.

Since the Bott-Taubes integral $T(W) \in H^{0}\left(T_{2 n}^{*}\right)$, applied to a point $h(K) \in \mathcal{H}_{2 n}$ coming from an $n$-singular knot $K$, is 0 in $H^{0}\left(T_{2 n-1}^{*}\right)$, we think of it as a map

$$
T(W): \mathcal{W}_{n} \longrightarrow H^{0}\left(T_{2 n}^{*}\right) / H^{0}\left(T_{2 n-1}^{*}\right),
$$




\section{VOLIĆ}

or, in other words, a map $\mathcal{W}_{n} \rightarrow E_{\infty}^{-2 n, 2 n}$ on $n$-singular knots. It thus fits the diagram (47) as follows.

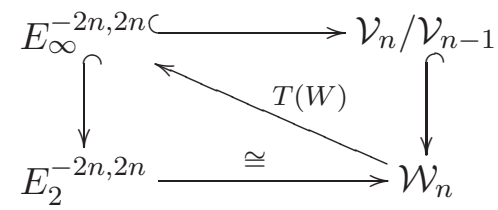

We have also modified the diagram by noticing that the top map has to be an injection because of the commutativity of the diagram.

However, now we can combine Theorem 3.3 and the fact from Theorem 4.2 that $W(D)$ restricts to a universal finite type invariant of ordinary knots. In particular, the following is easy to see.

Proposition 6.8. The composition

$$
\mathcal{W}_{n} \longrightarrow E_{\infty}^{-2 n, 2 n \hookrightarrow} \mathcal{V}_{n} / \mathcal{V}_{n-1} \longrightarrow \mathcal{W}_{n}
$$

is the identity.

The first observation now is that the map

$$
\mathcal{V}_{n} / \mathcal{V}_{n-1} \longrightarrow \mathcal{W}_{n}
$$

must be a surjection, so that we have obtained an alternative proof of the Kontsevich theorem.

TheOREM 6.9. $\mathcal{V}_{n} / \mathcal{V}_{n-1} \cong \mathcal{W}_{n}$.

We then also have

$$
H^{0}\left(T_{2 n}^{*}\right) / H^{0}\left(T_{2 n-1}^{*}\right)=E_{\infty}^{-2 n, 2 n} \cong \mathcal{V}_{n} / \mathcal{V}_{n-1}
$$

However, now we can compare two exact sequences as follows.

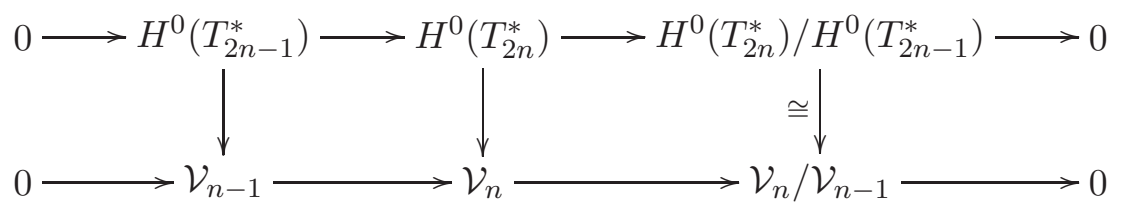

The first two vertical maps are duals to those given in (45).

Since the right square commutes, (50) can be used with $n=1$ as the base case for an induction which shows that the middle vertical map is an isomorphism if the left map is. We thus have the following.

THEOREM 6.10. $H^{0}\left(T_{2 n}^{*}\right) \cong \mathcal{V}_{n}$.

Finally, $E_{\infty}^{-2 n, 2 n C} \longrightarrow E_{2}^{-2 n, 2 n}$ also has to be an isomorphism since it is the last map in the commutative square with three other isomorphisms. We rephrase this as follows.

Theorem 6.11. The spectral sequence for the cohomology of $X^{\bullet}$ collapses at $E_{2}$ on the diagonal.

The preceding two results are precisely Theorems 1.2 and 1.3.

\subsection{Some further questions}

The Taylor tower for $\overline{\mathcal{K}}$ is thus a potentially rich source of information about finite type theory. Its advantage is that the stages $\mathcal{H}_{r}$ lend themselves to purely topological examination. At the time of writing, not much is known about these spaces, but our results give evidence that a closer look may shed some light on classical knot theory. In particular, the following questions should be addressed. 


\section{Finite TYPE KNOT INVARIANTS AND THE CALCULUS OF FUNCTORS}

- For knots in $\mathbb{R}^{n}, n>3$, the cohomology spectral sequence is guaranteed to converge to the totalization of $X^{\bullet}$. Combining this with the Goodwillie-Klein-Weiss result stating that in this case the Taylor tower converges to the space of knots, one can extract useful information about the homotopy and cohomology of spaces of knots [Sin02, LV05]. However, this may not be true in the case $n=3$ because it is not immediately clear that $C^{*}$ commutes with totalization. If the spectral sequence indeed does not converge to the desired associated graded, then potentially an even more interesting question arises: what can we say about invariants pulled back from the Taylor tower without passing to cochains first? It is quite possible that in this case one also obtains only finite type invariants, but the genuine Taylor tower for $\overline{\mathcal{K}}$ could also contain more information.

- Can one gain new insight into finite type theory by studying the homotopy types of spaces $\mathcal{H}_{r}$ ? In particular, can one gain topological insight into the common thread between knots in $\mathbb{R}^{3}$ and $\mathbb{R}^{n}$, namely the Kontsevich and Bott-Taubes integral constructions of finite type invariants? This is to be expected, since the latter type of integrals plays a crucial role in our proofs.

- What is the relationship between the Taylor tower and Vassiliev's approach to studying $\mathcal{K}$ through simplicial spaces [Vas92]?

- Do other finite type invariants (of homology spheres, for example) also factor through constructions coming from calculus of functors? One should at least be able to carry the ideas and constructions here from knots to braids without too much difficulty.

- Can one say anything useful about the inverse limit of the Taylor tower? The question of invariants of this limit surjecting onto knot invariants is precisely the question of finite type invariants separating knots.

- Recall that the evaluation map $e v^{*}$ provides the isomorphism of Theorem 6.10. Budney et al. [BCSS05] examine the evaluation map closely related to ours to give a geometric interpretation of the unique (up to framing) type 2 invariant. Can one in general understand the geometry of finite type invariants using the evaluation map?

- As predicted by a result in [GK05], is the map $\mathcal{K} \rightarrow \mathcal{H}_{r}$ 0-connected, i.e. surjective on $\pi_{0}$ ?

\section{ACKNOWLEDGEMENTS}

I am deeply endebted to my thesis advisor, Tom Goodwillie, whose knowledge is only matched by his desire to share it. Dev Sinha's help over the years has also been invaluable. I am grateful to Pascal Lambrechts and Greg Arone for many comments and suggestions.

\section{REFERENCES}

AF95 D. Altschuler and L. Freidel, On universal Vassiliev invariants, Comm. Math. Phys. 170 (1995), 41-62.

AS94 S. Axelrod and I. Singer, Chern-Simons perturbation theory, II, J. Differential Geom. 39 (1994), $173-213$.

Bar95 D. Bar-Natan, On the Vassiliev knot invariants, Topology 34 (1995), 423-472.

BS97 D. Bar-Natan and A. Stoimenow, The fundamental theorem of Vassiliev invariants, Lecture Notes in Pure and Applied Mathematics, vol. 184 (Springer, Berlin, 1997).

BT94 R. Bott and C. Taubes, On the self-linking of knots, J. Math. Phys. 35 (1994), 5247-5287.

Bou87 A. Bousfield, On the homology spectral sequence of a cosimplicial space, Amer. J. Math. 109 (1987), 361-394. 


\section{Finite TYPE KNOT INVARIANTS AND THE CALCULUS OF FUNCTORS}

BK72 A. Bousfield and D. Kan, Homotopy limits, completions, and localizations, Lecture Notes in Mathematics, vol. 304 (Springer, Berlin, 1972).

BCSS05 R. Budney, J. Conant, K. Scannell and D. Sinha, New perspectives on self-linking, Adv. Math. 191 (2005), 78-113.

Coh76 F. Cohen, The homology of $C_{n+1}$ spaces, Lecture Notes in Mathematics, vol. 533 (Springer, Berlin, 1976).

Dol58 A. Dold, Homology of symmetric products and other functors of complexes, Ann. of Math. (2) 68 (1958), 54-80.

DT58 A. Dold and R. Thom, Quasifaserungen und unendliche symmetrischen Produkte, Ann. of Math. (2) 67 (1958), 239-281.

FM94 W. Fulton and R. MacPherson, Compactification of configuration spaces, Ann. of Math. (2) 139 (1994), 183-225.

Goo90 T. Goodwillie, Calculus I: the first derivative of pseudoisotopy theory, K-Theory 4 (1990), 1-27.

Goo92 T. Goodwillie, Calculus II: analytic functors, K-Theory 5 (1991/92), 295-332.

Goo93 T. Goodwillie, Calculus III: Taylor series, Geom. Topol. 7 (2003), 645-711.

GK05 T. Goodwillie and J. Klein, Excision statements for spaces of embeddings, in preparation.

GKW01 T. Goodwillie, J. Klein, and M. Weiss, Spaces of smooth embeddings, disjunction and surgery, Surveys Surgery Theory $\mathbf{2}$ (2001), 221-284.

GW99 T. Goodwillie and M. Weiss, Embeddings from the point of view of immersion theory II, Geom. Topol. 3 (1999), 103-118.

Hat01 A. Hatcher, Algebraic topology (Cambridge University Press, Cambridge, 2001).

Kan58 D. Kan, Functors involving c.s.s. complexes, Trans. Amer. Math. Soc. 87 (1958), 330-346.

Kon93 M. Kontsevich, Vassiliev's knot invariants, Adv. Sov. Math. 16 (1993), 137-150.

Lab99 J. Labastida, Chern-Simons gauge theory: ten years after, in Trends in theoretical physics, II (Buenos Aires, 1998), AIP Conference Proceedings, vol. 484 (American Institute of Physics, Woodbury, NY, 1999).

LV05 P. Lambrechts and I. Volić, The rational homotopy type of spaces of knots in high codimension, in preparation.

May67 J. P. May, Simplicial objects in algebraic topology (Chicago University Press, Chicago, IL, 1967).

SS02 K. Scannell and D. Sinha, A one-dimensional embedding complex, J. Pure Appl. Algebra 170 (2002), 93-107.

Sin04a D. Sinha, Operads and knot spaces, Preprint (2004), math.AT/0407039.

Sin04b D. Sinha, Manifold-theoretic compactifications of configuration spaces, Selecta Math. (N.S.) 10 (2004), 391-428.

Sin02 D. Sinha, The topology of spaces of knots, Preprint (2003), math.AT/0202287.

Thu95 D. Thurston, Integral expressions for the Vassiliev knot invariants, Senior Thesis, Harvard University, 1995.

Vas92 V. Vassiliev, Complements of discriminants of smooth maps: topology and applications, Translations of Mathematical Monographs, vol. 98 (American Mathematical Society, Providence, RI, 1992).

Vol04 I. Volić, Configuration space integrals and Taylor towers for spaces of knots, Preprint (2004), math.GT/0401282.

Wei99 M. Weiss, Embeddings from the point of view of immersion theory I, Geom. Topol. 3 (1999), $67-101$.

Ismar Volić ismar@virginia.edu

Department of Mathematics, University of Virginia, Charlottesville, VA 22904-4137, USA 